\title{
A modified projection method for a common solution of a system of variational inequalities, a split equilibrium problem and a hierarchical fixed-point problem
}

\section{Abdellah Bnouhachem*}

"Correspondence:

babedallah@yahoo.com

School of Management Science and

Engineering, Nanjing University,

Nanjing, 210093, P.R. China

ENSA, Ibn Zohr University, Agadir,

BP 1136, Morocco

\section{Springer}

\begin{abstract}
In this paper, we suggest and analyze a modified projection method for finding a common solution of a system of variational inequalities, a split equilibrium problem, and a hierarchical fixed-point problem in the setting of real Hilbert spaces. We prove the strong convergence of the sequence generated by the proposed method to a common solution of a system of variational inequalities, a split equilibrium problem, and a hierarchical fixed-point problem. Several special cases are also discussed. The results presented in this paper extend and improve some well-known results in the literature.
\end{abstract}

MSC: 49J30; 47H09; 47J20

Keywords: system of variational inequalities; equilibrium problem; hierarchical fixed-point problem; fixed-point problem; projection method

\section{Introduction}

Let $H$ be a real Hilbert space, whose inner product and norm are denoted by $\langle\cdot, \cdot\rangle$ and $\|\cdot\|$. Let $C$ be a nonempty closed convex subset of $H$. Recently, Ceng et al. [1] considered the general system of variational inequalities, which involves finding $\left(x^{*}, y^{*}\right) \in C \times C$ such that

$$
\begin{cases}\left\langle\mu_{1} B_{1} y^{*}+x^{*}-y^{*}, x-x^{*}\right\rangle \geq 0 ; & \forall x \in C \text { and } \mu_{1}>0, \\ \left\langle\mu_{2} B_{2} x^{*}+y^{*}-x^{*}, x-y^{*}\right\rangle \geq 0 ; & \forall x \in C \text { and } \mu_{2}>0\end{cases}
$$

where $B_{i}: C \rightarrow C$ is a nonlinear mapping for each $i=1,2$. The solution set of (1.1) is denoted by $S^{*}$. As pointed out in [2] the system of variational inequalities is used as a tool to study the Nash equilibrium problem, see, for example, [3-6] and the references therein. We believe that the problem (1.1) could be used to study the Nash equilibrium problem for a two players game. The theory of variational inequalities is well established and it has a wide range of applications in science, engineering, management, and social sciences, see, for example, [4-7] and the references therein.

Ceng et al. [1] transformed problem (1.1) into a fixed-point problem (see Lemma 2.2) and introduced an iterative method for finding the common element of the set $\operatorname{Fix}(T) \cap S^{*}$. Based on the one-step iterative method [8] and the multi-step iterative method [9], Latif et al. [10] proposed a multi-step hybrid viscosity method that generates a sequence via

(C2014 Bnouhachem; licensee Springer. This is an Open Access article distributed under the terms of the Creative Commons Attribution License (http://creativecommons.org/licenses/by/2.0), which permits unrestricted use, distribution, and reproduction in any medium, provided the original work is properly cited. 
an explicit iterative algorithm to compute the approximate solutions of a system of variational inequalities defined over the intersection of the set of solutions of an equilibrium problem, the set of common fixed points of a finite family of nonexpansive mappings, and the solution set of a nonexpansive mapping. Under very mild conditions, they proved that the sequence converges strongly to a unique solution of system of variational inequalities defined over the set consisting of the set of solutions of an equilibrium problem, the set of common fixed points of nonexpansive mappings, and the set of fixed points of a mapping, and to a unique solution of the triple hierarchical variational inequality problem.

On the other hand, by combining the regularization method, Korpelevich's extragradient method, the hybrid steepest-descent method, and the viscosity approximation method, Ceng et al. [2] introduced and analyzed implicit and explicit iterative schemes for computing a common element of the solution set of system of variational inequalities and a set of zeros of an accretive operator in Banach space. Under suitable assumptions, they proved the strong convergence of the sequences generated by the proposed schemes.

If $B_{1}=B_{2}=B$, then the problem (1.1) reduces to finding $\left(x^{*}, y^{*}\right) \in C \times C$ such that

$$
\begin{cases}\left\langle\mu_{1} B y^{*}+x^{*}-y^{*}, x-x^{*}\right\rangle \geq 0 ; & \forall x \in C \text { and } \mu_{1}>0, \\ \left\langle\mu_{2} B x^{*}+y^{*}-x^{*}, x-y^{*}\right\rangle \geq 0 ; & \forall x \in C \text { and } \mu_{2}>0,\end{cases}
$$

which has been introduced and studied by Verma [11, 12].

If $x^{*}=y^{*}$ and $\mu_{1}=\mu_{2}$, then the problem (1.2) collapses to the classical variational inequality: finding $x^{*} \in C$, such that

$$
\left\langle B x^{*}, x-x^{*}\right\rangle \geq 0, \quad \forall x \in C .
$$

For the recent applications, numerical techniques, and physical formulation, see [1-45].

We introduce the following definitions, which are useful in the following analysis.

Definition 1.1 The mapping $T: C \rightarrow H$ is said to be

(a) monotone, if

$$
\langle T x-T y, x-y\rangle \geq 0, \quad \forall x, y \in C
$$

(b) strongly monotone, if there exists an $\alpha>0$ such that

$$
\langle T x-T y, x-y\rangle \geq \alpha\|x-y\|^{2}, \quad \forall x, y \in C ;
$$

(c) $\alpha$-inverse strongly monotone, if there exists an $\alpha>0$ such that

$$
\langle T x-T y, x-y\rangle \geq \alpha\|T x-T y\|^{2}, \quad \forall x, y \in C ;
$$

(d) nonexpansive, if

$$
\|T x-T y\| \leq\|x-y\|, \quad \forall x, y \in C
$$

(e) $k$-Lipschitz continuous, if there exists a constant $k>0$ such that

$$
\|T x-T y\| \leq k\|x-y\|, \quad \forall x, y \in C ;
$$


(f) a contraction on $C$, if there exists a constant $0 \leq k<1$ such that

$$
\|T x-T y\| \leq k\|x-y\|, \quad \forall x, y \in C
$$

It is easy to observe that every $\alpha$-inverse strongly monotone $T$ is monotone and Lipschitz continuous. It is well known that every nonexpansive operator $T: H \rightarrow H$ satisfies, for all $(x, y) \in H \times H$, the inequality

$$
\langle(x-T(x))-(y-T(y)), T(y)-T(x)\rangle \leq \frac{1}{2}\|(T(x)-x)-(T(y)-y)\|^{2},
$$

and therefore, we get, for all $(x, y) \in H \times \operatorname{Fix}(T)$,

$$
\langle x-T(x), y-T(x)\rangle \leq \frac{1}{2}\|T(x)-x\|^{2} .
$$

The fixed-point problem for the mapping $T$ is to find $x \in C$ such that

$$
T x=x
$$

We denote by $F(T)$ the set of solutions of (1.5). It is well known that $F(T)$ is closed and convex, and $P_{F}(T)$ is well defined.

The equilibrium problem, denoted by $E P$, is to find $x \in C$ such that

$$
F(x, y) \geq 0, \quad \forall y \in C
$$

The solution set of (1.6) is denoted by $E P(F)$. Numerous problems in physics, optimization, and economics reduce to finding a solution of $(1.6)$, see [25, 38]. In 1994, Censor and Elfving [19] introduced and studied the following split feasibility problem.

Let $C$ and $Q$ be nonempty closed convex subsets of the infinite-dimensional real Hilbert spaces $H_{1}$ and $H_{2}$, respectively, and let $A \in B\left(H_{1}, H_{2}\right)$, where $B\left(H_{1}, H_{2}\right)$ denotes the collection of all bounded linear operators from $H_{1}$ to $H_{2}$. The problem is to find $x^{*}$ such that

$$
x^{*} \in C \quad \text { such that } A x^{*} \in Q \text {. }
$$

Very recently, Ceng et al. [22] introduced and analyzed an extragradient method with regularization for finding a common element of the solution set of the split feasibility problem and the set of fixed points of a nonexpansive mapping $S$ in the setting of infinitedimensional Hilbert spaces. By combining Mann's iterative method and the extragradient method, Ceng et al. [21] proposed three different kinds of Mann type iterative methods for finding a common element of the solution set of the split feasibility problem and the set of fixed points of a nonexpansive mapping $S$ in the setting of infinite-dimensional Hilbert spaces.

Recently, Censor et al. [23] introduced a new variational inequality problem which we call the split variational inequality problem (SVIP). Let $H_{1}$ and $H_{2}$ be two real Hilbert spaces. Given the operators $f: H_{1} \rightarrow H_{1}$ and $g: H_{2} \rightarrow H_{2}$, a bounded linear operator $A$ : $H_{1} \rightarrow H_{2}$, and the nonempty, closed, and convex subsets $C \subseteq H_{1}$ and $Q \subseteq H_{2}$, the SVIP is formulated as follows: find a point $x^{*} \in C$ such that

$$
\left\langle f\left(x^{*}\right), x-x^{*}\right\rangle \geq 0 \quad \text { for all } x \in C
$$


and such that

$$
y^{*}=A x^{*} \in Q \quad \text { solves } \quad\left\langle g\left(y^{*}\right), y-y^{*}\right\rangle \geq 0 \quad \text { for all } y \in Q \text {. }
$$

In [36], Moudafi introduced an iterative method which can be regarded as an extension of the method given by Censor et al. [23] for the following split monotone variational inclusions:

Find $x^{*} \in H_{1} \quad$ such that $\quad 0 \in f\left(x^{*}\right)+B_{1}\left(x^{*}\right)$

and such that

$$
y^{*}=A x^{*} \in H_{2} \quad \text { solves } \quad 0 \in g\left(y^{*}\right)+B_{2}\left(y^{*}\right),
$$

where $B_{i}: H_{i} \rightarrow 2^{H_{i}}$ is a set-valued mapping for $i=1,2$. Later Byrne et al. [18] generalized and extended the work of Censor et al. [23] and Moudafi [36].

In this paper, we consider the following pair of equilibrium problems, called split equilibrium problems: Let $F_{1}: C \times C \rightarrow R$ and $F_{2}: Q \times Q \rightarrow R$ be nonlinear bifunctions and $A: H_{1} \rightarrow H_{2}$ be a bounded linear operator, then the split equilibrium problem (SEP) is to find $x^{*} \in C$ such that

$$
F_{1}\left(x^{*}, x\right) \geq 0, \quad \forall x \in C,
$$

and such that

$$
y^{*}=A x^{*} \in Q \quad \text { solves } \quad F_{2}\left(y^{*}, y\right) \geq 0, \quad \forall y \in Q .
$$

The solution set of SEP (1.9)-(1.10) is denoted by $\Lambda=\left\{p \in E P\left(F_{1}\right): A p \in E P\left(F_{2}\right)\right\}$.

Let $S: C \rightarrow H$ be a nonexpansive mapping. The following problem is called a hierarchical fixed-point problem: find $x \in F(T)$ such that

$$
\langle x-S x, y-x\rangle \geq 0, \quad \forall y \in F(T) .
$$

It is known that the hierarchical fixed-point problem (1.11) links with some monotone variational inequalities and convex programming problems; see [45]. Various methods have been proposed to solve the hierarchical fixed-point problem; see Mainge and Moudafi [34] and Cianciaruso et al. [26]. In 2010, Yao et al. [45] introduced the following strong convergence iterative algorithm to solve the problem (1.11):

$$
\begin{aligned}
& y_{n}=\beta_{n} S x_{n}+\left(1-\beta_{n}\right) x_{n}, \\
& x_{n+1}=P_{C}\left[\alpha_{n} f\left(x_{n}\right)+\left(1-\alpha_{n}\right) T y_{n}\right], \quad \forall n \geq 0,
\end{aligned}
$$

where $f: C \rightarrow H$ is a contraction mapping and $\left\{\alpha_{n}\right\}$ and $\left\{\beta_{n}\right\}$ are two sequences in $(0,1)$. Under some certain restrictions on the parameters, Yao et al. proved that the sequence $\left\{x_{n}\right\}$ generated by (1.12) converges strongly to $z \in F(T)$, which is the unique solution of the following variational inequality:

$$
\langle(I-f) z, y-z\rangle \geq 0, \quad \forall y \in F(T) .
$$


In 2011, Ceng et al. [20] investigated the following iterative method:

$$
x_{n+1}=P_{C}\left[\alpha_{n} \rho U\left(x_{n}\right)+\left(I-\alpha_{n} \mu F\right)\left(T\left(y_{n}\right)\right)\right], \quad \forall n \geq 0,
$$

where $U$ is a Lipschitzian mapping, and $F$ is a Lipschitzian and strongly monotone mapping. They proved that under some approximate assumptions on the operators and parameters, the sequence $\left\{x_{n}\right\}$ generated by (1.14) converges strongly to the unique solution of the variational inequality

$$
\langle\rho U(z)-\mu F(z), x-z\rangle \geq 0, \quad \forall x \in \operatorname{Fix}(T)
$$

In this paper, motivated by the work of Censor et al. [23], Moudafi [36], Byrne et al. [18], Yao et al. [45], Ceng et al. [20], Bnouhachem [15-17] and by the recent work going in this direction, we give an iterative method for finding the approximate element of the common set of solutions of (1.1), (1.9)-(1.10), and (1.11) in real Hilbert space. We establish a strong convergence theorem based on this method. We would like to mention that our proposed method is quite general and flexible and includes many known results for solving a system of variational inequality problems, split equilibrium problems, and hierarchical fixed-point problems, see, e.g. [20, 26, 34, 40, 45] and relevant references cited therein.

\section{Preliminaries}

In this section, we list some fundamental lemmas that are useful in the consequent analysis. The first lemma provides some basic properties of projection onto $C$.

Lemma 2.1 Let $P_{C}$ denote the projection of $H$ onto $C$. Then we have the following inequalities:

$$
\begin{aligned}
& \left\langle z-P_{C}[z], P_{C}[z]-v\right\rangle \geq 0, \quad \forall z \in H, v \in C ; \\
& \left\langle u-v, P_{C}[u]-P_{C}[v]\right\rangle \geq\left\|P_{C}[u]-P_{C}[v]\right\|^{2}, \quad \forall u, v \in H ; \\
& \left\|P_{C}[u]-P_{C}[v]\right\| \leq\|u-v\|, \quad \forall u, v \in H ; \\
& \left\|u-P_{C}[z]\right\|^{2} \leq\|z-u\|^{2}-\left\|z-P_{C}[z]\right\|^{2}, \quad \forall z \in H, u \in C .
\end{aligned}
$$

Lemma 2.2 [1] For any $\left(x^{*}, y^{*}\right) \in C \times C,\left(x^{*}, y^{*}\right)$ is a solution of (1.1) if and only if $x^{*}$ is a fixed point of the mapping $Q: C \rightarrow C$ defined by

$$
Q(x)=P_{C}\left[P_{C}\left[x-\mu_{2} B_{2} x\right]-\mu_{1} B_{1} P_{C}\left[x-\mu_{2} B_{2} x\right]\right], \quad \forall x \in C,
$$

where $y^{*}=P_{C}\left[x^{*}-\mu_{2} B_{2} x^{*}\right], \mu_{i} \in\left(0,2 \theta_{i}\right)$ and $B_{i}: C \rightarrow C$ is for the $\theta_{i}$-inverse strongly monotone mappings for each $i=1,2$.

Assumption 2.1 [14] Let $F: C \times C \rightarrow \mathbb{R}$ be a bifunction satisfying the following assumptions:

(i) $F(x, x)=0, \forall x \in C$;

(ii) $F$ is monotone, i.e., $F(x, y)+F(y, x) \leq 0, \forall x, y \in C$;

(iii) for each $x, y, z \in C, \lim _{t \rightarrow 0} F(t z+(1-t) x, y) \leq F(x, y)$; 
(iv) for each $x \in C, y \rightarrow F(x, y)$ is convex and lower semicontinuous;

(v) for fixed $r>0$ and $z \in C$, there exists a bounded subset $K$ of $H_{1}$ and $x \in C \cap K$ such that

$$
F(y, x)+\frac{1}{r}\langle y-x, x-z\rangle \geq 0, \quad \forall y \in C \backslash K .
$$

Lemma 2.3 [28] Assume that $F_{1}: C \times C \rightarrow \mathbb{R}$ satisfies Assumption 2.1. For $r>0$ and $\forall x \in$ $H_{1}$, define a mapping $T_{r}^{F_{1}}: H_{1} \rightarrow C$ as follows:

$$
T_{r}^{F_{1}}(x)=\left\{z \in C: F_{1}(z, y)+\frac{1}{r}\langle y-z, z-x\rangle \geq 0, \forall y \in C\right\} .
$$

Then the following hold:

(i) $T_{r}^{F_{1}}$ is nonempty and single-valued;

(ii) $T_{r}^{F_{1}}$ is firmly nonexpansive, i.e.,

$$
\left\|T_{r}^{F_{1}}(x)-T_{r}^{F_{1}}(y)\right\|^{2} \leq\left\langle T_{r}^{F_{1}}(x)-T_{r}^{F_{1}}(y), x-y\right\rangle, \quad \forall x, y \in H_{1}
$$

(iii) $F\left(T_{r}^{F_{1}}\right)=E P\left(F_{1}\right)$;

(iv) $E P\left(F_{1}\right)$ is closed and convex.

Assume that $F_{2}: Q \times Q \rightarrow \mathbb{R}$ satisfies Assumption 2.1, and for $s>0$ and $\forall u \in H_{2}$, define a mapping $T_{s}^{F_{2}}: H_{2} \rightarrow Q$ as follows:

$$
T_{s}^{F_{2}}(u)=\left\{v \in Q: F_{2}(v, w)+\frac{1}{s}\langle w-v, v-u\rangle \geq 0, \forall w \in Q\right\} .
$$

Then $T_{s}^{F_{2}}$ satisfies conditions (i)-(iv) of Lemma 2.3. $F\left(T_{s}^{F_{2}}\right)=E P\left(F_{2}, Q\right)$, where $E P\left(F_{2}, Q\right)$ is the solution set of the following equilibrium problem:

Find $y^{*} \in Q \quad$ such that $\quad F_{2}\left(y^{*}, y\right) \geq 0, \quad \forall y \in Q$.

Lemma 2.4 [27] Assume that $F_{1}: C \times C \rightarrow \mathbb{R}$ satisfies Assumption 2.1, and let $T_{r}^{F_{1}}$ be defined as in Lemma 2.3. Let $x, y \in H_{1}$ and $r_{1}, r_{2}>0$. Then

$$
\left\|T_{r_{2}}^{F_{1}}(y)-T_{r_{1}}^{F_{1}}(x)\right\| \leq\|y-x\|+\left|\frac{r_{2}-r_{1}}{r_{2}}\right|\left\|T_{r_{2}}^{F_{1}}(y)-y\right\| .
$$

Lemma 2.5 [31] Let C be a nonempty closed convex subset of a real Hilbert space H. If $T$ : $C \rightarrow C$ is a nonexpansive mapping with $\operatorname{Fix}(T) \neq \emptyset$, then the mapping $I-T$ is demiclosed at 0 , i.e., if $\left\{x_{n}\right\}$ is a sequence in $C$ weakly converges to $x$ and if $\left\{(I-T) x_{n}\right\}$ converges strongly to 0 , then $(I-T) x=0$.

Lemma 2.6 [20] Let $U: C \rightarrow H$ be a $\tau$-Lipschitzian mapping and let $F: C \rightarrow H$ be a $k$-Lipschitzian and $\eta$-strongly monotone mapping, then for $0 \leq \rho \tau<\mu \eta, \mu F-\rho U$ is $\mu \eta-$ $\rho \tau$-strongly monotone i.e.,

$$
\langle(\mu F-\rho U) x-(\mu F-\rho U) y, x-y\rangle \geq(\mu \eta-\rho \tau)\|x-y\|^{2}, \quad \forall x, y \in C .
$$


Lemma 2.7 [39] Suppose that $\lambda \in(0,1)$ and $\mu>0$. Let $F: C \rightarrow H$ be a $k$-Lipschitzian and $\eta$-strongly monotone operator. In association with a nonexpansive mapping $T: C \rightarrow C$, define the mapping $T^{\lambda}: C \rightarrow H$ by

$$
T^{\lambda} x=T x-\lambda \mu F T(x), \quad \forall x \in C .
$$

Then $T^{\lambda}$ is a contraction provided $\mu<\frac{2 \eta}{k^{2}}$, that is,

$$
\left\|T^{\lambda} x-T^{\lambda} y\right\| \leq(1-\lambda v)\|x-y\|, \quad \forall x, y \in C,
$$

where $v=1-\sqrt{1-\mu\left(2 \eta-\mu k^{2}\right)}$.

Lemma 2.8 [43] Assume $\left\{a_{n}\right\}$ is a sequence of nonnegative real numbers such that

$$
a_{n+1} \leq\left(1-\gamma_{n}\right) a_{n}+\delta_{n}
$$

where $\left\{\gamma_{n}\right\}$ is a sequence in $(0,1)$ and $\delta_{n}$ is a sequence such that

(1) $\sum_{n=1}^{\infty} \gamma_{n}=\infty$;

(2) $\lim \sup _{n \rightarrow \infty} \delta_{n} / \gamma_{n} \leq 0$ or $\sum_{n=1}^{\infty}\left|\delta_{n}\right|<\infty$.

Then $\lim _{n \rightarrow \infty} a_{n}=0$.

Lemma 2.9 [13] Let $C$ be a closed convex subset of $H$. Let $\left\{x_{n}\right\}$ be a bounded sequence in H. Assume that

(i) the weak $w$-limit set $w_{w}\left(x_{n}\right) \subset C$ where $w_{w}\left(x_{n}\right)=\left\{x: x_{n_{i}} \rightarrow x\right\}$;

(ii) for each $z \in C, \lim _{n \rightarrow \infty}\left\|x_{n}-z\right\|$ exists.

Then $\left\{x_{n}\right\}$ is weakly convergent to a point in $C$.

\section{The proposed method and some properties}

In this section, we suggest and analyze our method for finding the common solutions of the system of the variational inequality problem (1.1), the split equilibrium problem (1.9)(1.10), and the hierarchical fixed-point problem (1.11).

Let $H_{1}$ and $H_{2}$ be two real Hilbert spaces and $C \subseteq H_{1}$ and $Q \subseteq H_{2}$ be nonempty closed convex subsets of Hilbert spaces $H_{1}$ and $H_{2}$, respectively. Let $A: H_{1} \rightarrow H_{2}$ be a bounded linear operator. Assume that $F_{1}: C \times C \rightarrow \mathbb{R}$ and $F_{2}: Q \times Q \rightarrow \mathbb{R}$ are the bifunctions satisfying Assumption 2.1 and $F_{2}$ is upper semicontinuous in the first argument. Let $B_{i}$ : $C \rightarrow H$ be a $\theta_{i}$-inverse strongly monotone mapping for each $i=1,2$ and $S, T: C \rightarrow C$ a nonexpansive mappings such that $S^{*} \cap \Lambda \cap F(T) \neq \emptyset$. Let $F: C \rightarrow C$ be a $k$-Lipschitzian mapping and be $\eta$-strongly monotone, and let $U: C \rightarrow C$ be a $\tau$-Lipschitzian mapping.

Algorithm 3.1 For an arbitrary given $x_{0} \in C$, let the iterative sequences $\left\{u_{n}\right\},\left\{x_{n}\right\},\left\{y_{n}\right\}$, and $\left\{z_{n}\right\}$ be generated by

$$
\left\{\begin{array}{l}
u_{n}=T_{r_{n}}^{F_{1}}\left(x_{n}+\gamma A^{*}\left(T_{r_{n}}^{F_{2}}-I\right) A x_{n}\right) ; \\
z_{n}=P_{C}\left[P_{C}\left[u_{n}-\mu_{2} B_{2} u_{n}\right]-\mu_{1} B_{1} P_{C}\left[u_{n}-\mu_{2} B_{2} u_{n}\right]\right] ; \\
y_{n}=\beta_{n} S x_{n}+\left(1-\beta_{n}\right) z_{n} ; \\
x_{n+1}=P_{C}\left[\alpha_{n} \rho U\left(x_{n}\right)+\left(I-\alpha_{n} \mu F\right)\left(T\left(y_{n}\right)\right)\right], \quad \forall n \geq 0,
\end{array}\right.
$$

where $\mu_{i} \in\left(0,2 \theta_{i}\right)$ for each $i=1,2,\left\{r_{n}\right\} \subset(0,2 \varsigma)$ and $\gamma \in(0,1 / L), L$ is the spectral radius of the operator $A^{*} A$, and $A^{*}$ is the adjoint of $A$. Suppose the parameters satisfy $0<\mu<\frac{2 \eta}{k^{2}}$, 
$0 \leq \rho \tau<\nu$, where $v=1-\sqrt{1-\mu\left(2 \eta-\mu k^{2}\right)}$. Also, $\left\{\alpha_{n}\right\}$ and $\left\{\beta_{n}\right\}$ are sequences in $(0,1)$ satisfying the following conditions:

(a) $\lim _{n \rightarrow \infty} \alpha_{n}=0$ and $\sum_{n=1}^{\infty} \alpha_{n}=\infty$,

(b) $\lim _{n \rightarrow \infty}\left(\beta_{n} / \alpha_{n}\right)=0$,

(c) $\sum_{n=1}^{\infty}\left|\alpha_{n-1}-\alpha_{n}\right|<\infty$ and $\sum_{n=1}^{\infty}\left|\beta_{n-1}-\beta_{n}\right|<\infty$,

(d) $\liminf _{n \rightarrow \infty} r_{n}<\limsup _{n \rightarrow \infty} r_{n}<2 \varsigma$ and $\sum_{n=1}^{\infty}\left|r_{n-1}-r_{n}\right|<\infty$.

Remark 3.1 Our method can be viewed as an extension and improvement for some wellknown results, for example the following.

- The proposed method is an extension and improvement of the method of Wang and $\mathrm{Xu}$ [42] for finding the approximate element of the common set of solutions of a split equilibrium problem and a hierarchical fixed-point problem in a real Hilbert space.

- If we have the Lipschitzian mapping $U=f, F=I, \rho=\mu=1$, and $B_{1}=B_{2}=0$, we obtain an extension and improvement of the method of Yao et al. [45] for finding the approximate element of the common set of solutions of a split equilibrium problem and a hierarchical fixed-point problem in a real Hilbert space.

- The contractive mapping $f$ with a coefficient $\alpha \in[0,1)$ in other papers $[39,40,45]$ is extended to the cases of the Lipschitzian mapping $U$ with a coefficient constant $\gamma \in[0, \infty)$.

This shows that Algorithm 3.1 is quite general and unifying.

Lemma 3.1 Let $x^{*} \in S^{*} \cap \Lambda \cap F(T)$. Then $\left\{x_{n}\right\},\left\{u_{n}\right\},\left\{z_{n}\right\}$, and $\left\{y_{n}\right\}$ are bounded.

Proof Let $x^{*} \in S^{*} \cap \Lambda \cap F(T)$; we have $x^{*}=T_{r_{n}}^{F_{1}}\left(x^{*}\right)$ and $A x^{*}=T_{r_{n}}^{F_{2}}\left(A x^{*}\right)$. Then

$$
\begin{aligned}
\left\|u_{n}-x^{*}\right\|^{2}= & \left\|T_{r_{n}}^{F_{1}}\left(x_{n}+\gamma A^{*}\left(T_{r_{n}}^{F_{2}}-I\right) A x_{n}\right)-x^{*}\right\|^{2} \\
= & \left\|T_{r_{n}}^{F_{1}}\left(x_{n}+\gamma A^{*}\left(T_{r_{n}}^{F_{2}}-I\right) A x_{n}\right)-T_{r_{n}}^{F_{1}}\left(x^{*}\right)\right\|^{2} \\
\leq & \left\|x_{n}+\gamma A^{*}\left(T_{r_{n}}^{F_{2}}-I\right) A x_{n}-x^{*}\right\|^{2} \\
= & \left\|x_{n}-x^{*}\right\|^{2}+\gamma^{2}\left\|A^{*}\left(T_{r_{n}}^{F_{2}}-I\right) A x_{n}\right\|^{2}+2 \gamma\left\langle x_{n}-x^{*}, A^{*}\left(T_{r_{n}}^{F_{2}}-I\right) A x_{n}\right\rangle \\
= & \left\|x_{n}-x^{*}\right\|^{2}+\gamma^{2}\left\langle\left(T_{r_{n}}^{F_{2}}-I\right) A x_{n}, A A^{*}\left(T_{r_{n}}^{F_{2}}-I\right) A x_{n}\right\rangle \\
& +2 \gamma\left\langle x_{n}-x^{*}, A^{*}\left(T_{r_{n}}^{F_{2}}-I\right) A x_{n}\right\rangle .
\end{aligned}
$$

From the definition of $L$ it follows that

$$
\begin{aligned}
\gamma^{2}\left\langle\left(T_{r_{n}}^{F_{2}}-I\right) A x_{n}, A A^{*}\left(T_{r_{n}}^{F_{2}}-I\right) A x_{n}\right\rangle & \leq L \gamma^{2}\left\langle\left(T_{r_{n}}^{F_{2}}-I\right) A x_{n},\left(T_{r_{n}}^{F_{2}}-I\right) A x_{n}\right\rangle \\
& =L \gamma^{2}\left\|\left(T_{r_{n}}^{F_{2}}-I\right) A x_{n}\right\|^{2} .
\end{aligned}
$$

It follows from (1.4) that

$$
\begin{aligned}
2 \gamma & \left\langle x_{n}-x^{*}, A^{*}\left(T_{r_{n}}^{F_{2}}-I\right) A x_{n}\right\rangle \\
& =2 \gamma\left\langle A\left(x_{n}-x^{*}\right),\left(T_{r_{n}}^{F_{2}}-I\right) A x_{n}\right\rangle \\
& =2 \gamma\left\langle A\left(x_{n}-x^{*}\right)+\left(T_{r_{n}}^{F_{2}}-I\right) A x_{n}-\left(T_{r_{n}}^{F_{2}}-I\right) A x_{n},\left(T_{r_{n}}^{F_{2}}-I\right) A x_{n}\right\rangle \\
& =2 \gamma\left(\left\langle T_{r_{n}}^{F_{2}} A x_{n}-A x^{*},\left(T_{r_{n}}^{F_{2}}-I\right) A x_{n}\right\rangle-\left\|\left(T_{r_{n}}^{F_{2}}-I\right) A x_{n}\right\|^{2}\right)
\end{aligned}
$$




$$
\begin{aligned}
& \leq 2 \gamma\left(\frac{1}{2}\left\|\left(T_{r_{n}}^{F_{2}}-I\right) A x_{n}\right\|^{2}-\left\|\left(T_{r_{n}}^{F_{2}}-I\right) A x_{n}\right\|^{2}\right) \\
& =-\gamma\left\|\left(T_{r_{n}}^{F_{2}}-I\right) A x_{n}\right\|^{2} .
\end{aligned}
$$

Applying (3.4) and (3.3) to (3.2) and from the definition of $\gamma$, we get

$$
\begin{aligned}
\left\|u_{n}-x^{*}\right\|^{2} & \leq\left\|x_{n}-x^{*}\right\|^{2}+\gamma(L \gamma-1)\left\|\left(T_{r_{n}}^{F_{2}}-I\right) A x_{n}\right\|^{2} \\
& \leq\left\|x_{n}-x^{*}\right\|^{2} .
\end{aligned}
$$

Let $x^{*} \in S^{*} \cap \Lambda \cap F(T)$; we have

$$
x^{*}=P_{C}\left[y^{*}-\mu_{1} B_{1} y^{*}\right]
$$

where

$$
y^{*}=P_{C}\left[x^{*}-\mu_{2} B_{2} x^{*}\right]
$$

We set $v_{n}=P_{C}\left[u_{n}-\mu_{2} B_{2} u_{n}\right]$. Since $B_{2}$ is a $\theta_{2}$-inverse strongly monotone mapping, it follows that

$$
\begin{aligned}
\left\|v_{n}-y^{*}\right\|^{2} & =\left\|P_{C}\left[u_{n}-\mu_{2} B_{2} u_{n}\right]-P_{C}\left[x^{*}-\mu_{2} B_{2} x^{*}\right]\right\|^{2} \\
& \leq\left\|u_{n}-x^{*}-\mu_{2}\left(B_{2} u_{n}-B_{2} x^{*}\right)\right\|^{2} \\
& \leq\left\|x_{n}-x^{*}\right\|^{2}-\mu_{2}\left(2 \theta_{2}-\mu_{2}\right)\left\|B_{2} u_{n}-B_{2} x^{*}\right\|^{2} \\
& \leq\left\|x_{n}-x^{*}\right\|^{2} .
\end{aligned}
$$

Since $B_{i}$ is $\theta_{i}$-inverse strongly monotone mappings, for each $i=1,2$, we get

$$
\begin{aligned}
\left\|z_{n}-x^{*}\right\|^{2}= & \| P_{C}\left[P_{C}\left[u_{n}-\mu_{2} B_{2} u_{n}\right]-\mu_{1} B_{1} P_{C}\left[u_{n}-\mu_{2} B_{2} u_{n}\right]\right] \\
& -P_{C}\left[P_{C}\left[x^{*}-\mu_{2} B_{2} x^{*}\right]-\mu_{1} B_{1} P_{C}\left[x^{*}-\mu_{2} B_{2} x^{*}\right]\right] \|^{2} \\
\leq & \| P_{C}\left[u_{n}-\mu_{2} B_{2} u_{n}\right]-\mu_{1} B_{1} P_{C}\left[u_{n}-\mu_{2} B_{2} u_{n}\right] \\
& -\left(P_{C}\left[x^{*}-\mu_{2} B_{2} x^{*}\right]-\mu_{1} B_{1} P_{C}\left[x^{*}-\mu_{2} B_{2} x^{*}\right]\right) \|^{2} \\
= & \| P_{C}\left[u_{n}-\mu_{2} B_{2} u_{n}\right]-P_{C}\left[x^{*}-\mu_{2} B_{2} x^{*}\right] \\
& -\mu_{1}\left(B_{1} P_{C}\left[u_{n}-\mu_{2} B_{2} u_{n}\right]-B_{1} P_{C}\left[x^{*}-\mu_{2} B_{2} x^{*}\right]\right) \|^{2} \\
\leq & \left\|P_{C}\left[u_{n}-\mu_{2} B_{2} u_{n}\right]-P_{C}\left[x^{*}-\mu_{2} B_{2} x^{*}\right]\right\|^{2} \\
& -\mu_{1}\left(2 \theta_{1}-\mu_{1}\right)\left\|B_{1} P_{C}\left[u_{n}-\mu_{2} B_{2} u_{n}\right]-B_{1} P_{C}\left[x^{*}-\mu_{2} B_{2} x^{*}\right]\right\|^{2} \\
\leq & \left\|\left(u_{n}-\mu_{2} B_{2} u_{n}\right)-\left(x^{*}-\mu_{2} B_{2} x^{*}\right)\right\|^{2} \\
& -\mu_{1}\left(2 \theta_{1}-\mu_{1}\right)\left\|B_{1} P_{C}\left[u_{n}-\mu_{2} B_{2} u_{n}\right]-B_{1} P_{C}\left[x^{*}-\mu_{2} B_{2} x^{*}\right]\right\|^{2} \\
\leq & \left\|u_{n}-x^{*}\right\|^{2}-\mu_{2}\left(2 \theta_{2}-\mu_{2}\right)\left\|B_{2} u_{n}-B_{2} x^{*}\right\|^{2}-\mu_{1}\left(2 \theta_{1}-\mu_{1}\right)\left\|B_{1} v_{n}-B_{1} y^{*}\right\|^{2} \\
\leq & \left\|u_{n}-x^{*}\right\|^{2} \\
\leq & \left\|x_{n}-x^{*}\right\|^{2} .
\end{aligned}
$$


We denote $V_{n}=\alpha_{n} \rho U\left(x_{n}\right)+\left(I-\alpha_{n} \mu F\right)\left(T\left(y_{n}\right)\right)$. Next, we prove that the sequence $\left\{x_{n}\right\}$ is bounded, and without loss of generality we can assume that $\beta_{n} \leq \alpha_{n}$ for all $n \geq 1$. From (3.1), we have

$$
\begin{aligned}
\left\|x_{n+1}-x^{*}\right\|= & \left\|P_{C}\left[V_{n}\right]-P_{C}\left[x^{*}\right]\right\| \\
\leq & \left\|\alpha_{n} \rho U\left(x_{n}\right)+\left(I-\alpha_{n} \mu F\right)\left(T\left(y_{n}\right)\right)-x^{*}\right\| \\
\leq & \alpha_{n}\left\|\rho U\left(x_{n}\right)-\mu F\left(x^{*}\right)\right\|+\left\|\left(I-\alpha_{n} \mu F\right)\left(T\left(y_{n}\right)\right)-\left(I-\alpha_{n} \mu F\right) T\left(x^{*}\right)\right\| \\
= & \alpha_{n}\left\|\rho U\left(x_{n}\right)-\rho U\left(x^{*}\right)+(\rho U-\mu F) x^{*}\right\| \\
& +\left\|\left(I-\alpha_{n} \mu F\right)\left(T\left(y_{n}\right)\right)-\left(I-\alpha_{n} \mu F\right) T\left(x^{*}\right)\right\| \\
\leq & \alpha_{n} \rho \tau\left\|x_{n}-x^{*}\right\|+\alpha_{n}\left\|(\rho U-\mu F) x^{*}\right\|+\left(1-\alpha_{n} v\right)\left\|y_{n}-x^{*}\right\| \\
\leq & \alpha_{n} \rho \tau\left\|x_{n}-x^{*}\right\|+\alpha_{n}\left\|(\rho U-\mu F) x^{*}\right\| \\
& +\left(1-\alpha_{n} v\right)\left\|\beta_{n} S x_{n}+\left(1-\beta_{n}\right) z_{n}-x^{*}\right\| \\
\leq & \alpha_{n} \rho \tau\left\|x_{n}-x^{*}\right\|+\alpha_{n}\left\|(\rho U-\mu F) x^{*}\right\|+\left(1-\alpha_{n} v\right)\left(\beta_{n}\left\|S x_{n}-S x^{*}\right\|\right. \\
& \left.+\beta_{n}\left\|S x^{*}-x^{*}\right\|+\left(1-\beta_{n}\right)\left\|z_{n}-x^{*}\right\|\right) \\
\leq & \alpha_{n} \rho \tau\left\|x_{n}-x^{*}\right\|+\alpha_{n}\left\|(\rho U-\mu F) x^{*}\right\|+\left(1-\alpha_{n} v\right)\left(\beta_{n}\left\|S x_{n}-S x^{*}\right\|\right. \\
& \left.+\beta_{n}\left\|S x^{*}-x^{*}\right\|+\left(1-\beta_{n}\right)\left\|x_{n}-x^{*}\right\|\right) \\
\leq & \alpha_{n} \rho \tau\left\|x_{n}-x^{*}\right\|+\alpha_{n}\left\|(\rho U-\mu F) x^{*}\right\|+\left(1-\alpha_{n} v\right)\left(\beta_{n}\left\|x_{n}-x^{*}\right\|\right. \\
& \left.+\beta_{n}\left\|S x^{*}-x^{*}\right\|+\left(1-\beta_{n}\right)\left\|x_{n}-x^{*}\right\|\right) \\
= & \left(1-\alpha_{n}(v-\rho \tau)\right)\left\|x_{n}-x^{*}\right\|+\alpha_{n}\left\|(\rho U-\mu F) x^{*}\right\| \\
& +\left(1-\alpha_{n} v\right) \beta_{n}\left\|S x^{*}-x^{*}\right\| \\
\leq & \left(1-\alpha_{n}(v-\rho \tau)\right)\left\|x_{n}-x^{*}\right\|+\alpha_{n}\left\|(\rho U-\mu F) x^{*}\right\|+\beta_{n}\left\|S x^{*}-x^{*}\right\| \\
\leq & \left(1-\alpha_{n}(v-\rho \tau)\right)\left\|x_{n}-x^{*}\right\|+\alpha_{n}\left(\left\|(\rho U-\mu F) x^{*}\right\|+\left\|S x^{*}-x^{*}\right\|\right) \\
= & \left(1-\alpha_{n}(v-\rho \tau)\right)\left\|x_{n}-x^{*}\right\| \\
& +\frac{\alpha_{n}(v-\rho \tau)}{v-\rho \tau}\left(\left\|(\rho U-\mu F) x^{*}\right\|+\left\|S x^{*}-x^{*}\right\|\right) \\
\leq & \max \left\{\left\|x_{n}-x^{*}\right\|, \frac{1}{v-\rho \tau}\left(\left\|(\rho U-\mu F) x^{*}\right\|+\left\|S x^{*}-x^{*}\right\|\right)\right\} \\
& \\
&
\end{aligned}
$$

where the third inequality follows from Lemma 2.7.

By induction on $n$, we obtain $\left\|x_{n}-x^{*}\right\| \leq \max \left\{\left\|x_{0}-x^{*}\right\|, \frac{1}{v-\rho \tau}\left(\left\|(\rho U-\mu F) x^{*}\right\|+\| S x^{*}-\right.\right.$ $\left.\left.x^{*} \|\right)\right\}$, for $n \geq 0$ and $x_{0} \in C$. Hence $\left\{x_{n}\right\}$ is bounded and, consequently, we deduce that $\left\{u_{n}\right\}$, $\left\{z_{n}\right\},\left\{v_{n}\right\},\left\{y_{n}\right\},\left\{S\left(x_{n}\right)\right\},\left\{T\left(x_{n}\right)\right\},\left\{F\left(T\left(y_{n}\right)\right)\right\}$, and $\left\{U\left(x_{n}\right)\right\}$ are bounded.

Lemma 3.2 Let $x^{*} \in S^{*} \cap \Lambda \cap F(T)$ and $\left\{x_{n}\right\}$ be the sequence generated by Algorithm 3.1. Then we have:

(a) $\lim _{n \rightarrow \infty}\left\|x_{n+1}-x_{n}\right\|=0$.

(b) The weak w-limit set $w_{w}\left(x_{n}\right) \subset F(T)\left(w_{w}\left(x_{n}\right)=\left\{x: x_{n_{i}} \rightarrow x\right\}\right)$. 
Proof Since $u_{n}=T_{r_{n}}^{F_{1}}\left(x_{n}+\gamma A^{*}\left(T_{r_{n}}^{F_{2}}-I\right) A x_{n}\right)$ and $u_{n-1}=T_{r_{n-1}}^{F_{1}}\left(x_{n-1}+\gamma A^{*}\left(T_{r_{n-1}}^{F_{2}}-I\right) A x_{n-1}\right)$. It follows from Lemma 2.4 that

$$
\begin{aligned}
& \left\|u_{n}-u_{n-1}\right\| \leq\left\|x_{n}-x_{n-1}+\gamma\left(A^{*}\left(T_{r_{n}}^{F_{2}}-I\right) A x_{n}-A^{*}\left(T_{r_{n-1}}^{F_{2}}-I\right) A x_{n-1}\right)\right\| \\
& +\left|1-\frac{r_{n-1}}{r_{n}}\right|\left\|T_{r_{n}}^{F_{1}}\left(x_{n}+\gamma A^{*}\left(T_{r_{n}}^{F_{2}}-I\right) A x_{n}\right)-\left(x_{n}+\gamma A^{*}\left(T_{r_{n}}^{F_{2}}-I\right) A x_{n}\right)\right\| \\
& \leq\left\|x_{n}-x_{n-1}-\gamma A^{*} A\left(x_{n}-x_{n-1}\right)\right\|+\gamma\|A\|\left\|T_{r_{n}}^{F_{2}} A x_{n}-T_{r_{n-1}}^{F_{2}} A x_{n-1}\right\| \\
& +\left|1-\frac{r_{n-1}}{r_{n}}\right|\left\|T_{r_{n}}^{F_{1}}\left(x_{n}+\gamma A^{*}\left(T_{r_{n}}^{F_{2}}-I\right) A x_{n}\right)-\left(x_{n}+\gamma A^{*}\left(T_{r_{n}}^{F_{2}}-I\right) A x_{n}\right)\right\| \\
& \leq\left(\left\|x_{n}-x_{n-1}\right\|^{2}-2 \gamma\left\|A\left(x_{n}-x_{n-1}\right)\right\|^{2}+\gamma^{2}\|A\|^{4}\left\|x_{n}-x_{n-1}\right\|^{2}\right)^{\frac{1}{2}} \\
& +\gamma\|A\|\left(\left\|A\left(x_{n}-x_{n-1}\right)\right\|+\left|1-\frac{r_{n-1}}{r_{n}}\right|\left\|T_{r_{n}}^{F_{2}} A x_{n}-A x_{n}\right\|\right) \\
& +\left|1-\frac{r_{n-1}}{r_{n}}\right|\left\|T_{r_{n}}^{F_{1}}\left(x_{n}+\gamma A^{*}\left(T_{r_{n}}^{F_{2}}-I\right) A x_{n}\right)-\left(x_{n}+\gamma A^{*}\left(T_{r_{n}}^{F_{2}}-I\right) A x_{n}\right)\right\| \\
& \leq\left(1-2 \gamma\|A\|^{2}+\gamma^{2}\|A\|^{4}\right)^{\frac{1}{2}}\left\|x_{n}-x_{n-1}\right\|+\gamma\|A\|^{2}\left\|x_{n}-x_{n-1}\right\| \\
& +\gamma\|A\|\left|1-\frac{r_{n-1}}{r_{n}}\right|\left\|T_{r_{n}}^{F_{2}} A x_{n}-A x_{n}\right\| \\
& +\left|1-\frac{r_{n-1}}{r_{n}}\right|\left\|T_{r_{n}}^{F_{1}}\left(x_{n}+\gamma A^{*}\left(T_{r_{n}}^{F_{2}}-I\right) A x_{n}\right)-\left(x_{n}+\gamma A^{*}\left(T_{r_{n}}^{F_{2}}-I\right) A x_{n}\right)\right\| \\
& =\left(1-\gamma\|A\|^{2}\right)\left\|x_{n}-x_{n-1}\right\|+\gamma\|A\|^{2}\left\|x_{n}-x_{n-1}\right\| \\
& +\gamma\|A\|\left|1-\frac{r_{n-1}}{r_{n}}\right|\left\|T_{r_{n}}^{F_{2}} A x_{n}-A x_{n}\right\| \\
& +\left|1-\frac{r_{n-1}}{r_{n}}\right|\left\|T_{r_{n}}^{F_{1}}\left(x_{n}+\gamma A^{*}\left(T_{r_{n}}^{F_{2}}-I\right) A x_{n}\right)-\left(x_{n}+\gamma A^{*}\left(T_{r_{n}}^{F_{2}}-I\right) A x_{n}\right)\right\| \\
& =\left\|x_{n}-x_{n-1}\right\|+\gamma\|A\|\left|1-\frac{r_{n-1}}{r_{n}}\right|\left\|T_{r_{n}}^{F_{2}} A x_{n}-A x_{n}\right\| \\
& +\left|1-\frac{r_{n-1}}{r_{n}}\right|\left\|T_{r_{n}}^{F_{1}}\left(x_{n}+\gamma A^{*}\left(T_{r_{n}}^{F_{2}}-I\right) A x_{n}\right)-\left(x_{n}+\gamma A^{*}\left(T_{r_{n}}^{F_{2}}-I\right) A x_{n}\right)\right\| \\
& =\left\|x_{n}-x_{n-1}\right\|+\left|\frac{r_{n}-r_{n-1}}{r_{n}}\right|\left(\gamma\|A\| \sigma_{n}+\chi_{n}\right) \text {, }
\end{aligned}
$$

where $\sigma_{n}:=\left\|T_{r_{n}}^{F_{2}} A x_{n}-A x_{n}\right\|$ and $\chi_{n}:=\| T_{r_{n}}^{F_{1}}\left(x_{n}+\gamma A^{*}\left(T_{r_{n}}^{F_{2}}-I\right) A x_{n}\right)-\left(x_{n}+\gamma A^{*}\left(T_{r_{n}}^{F_{2}}-\right.\right.$ I) $\left.A x_{n}\right) \|$. Without loss of generality, let us assume that there exists a real number $\mu$ such that $r_{n}>\mu>0$, for all positive integers $n$. Then we get

$$
\left\|u_{n-1}-u_{n}\right\| \leq\left\|x_{n-1}-x_{n}\right\|+\frac{1}{\mu}\left|r_{n-1}-r_{n}\right|\left(\gamma\|A\| \sigma_{n}+\chi_{n}\right) .
$$

Next, we estimate

$$
\begin{aligned}
\left\|z_{n}-z_{n-1}\right\|^{2}= & \| P_{C}\left[P_{C}\left[u_{n}-\mu_{2} B_{2} u_{n}\right]-\mu_{1} B_{1} P_{C}\left[u_{n}-\mu_{2} B_{2} u_{n}\right]\right] \\
& -P_{C}\left[P_{C}\left[u_{n-1}-\mu_{2} B_{2} u_{n-1}\right]-\mu_{1} B_{1} P_{C}\left[u_{n-1}-\mu_{2} B_{2} u_{n-1}\right]\right] \|^{2} \\
\leq & \| P_{C}\left[u_{n}-\mu_{2} B_{2} u_{n}\right]-\mu_{1} B_{1} P_{C}\left[u_{n}-\mu_{2} B_{2} u_{n}\right]
\end{aligned}
$$




$$
\begin{aligned}
& -\left(P_{C}\left[u_{n-1}-\mu_{2} B_{2} u_{n-1}\right]-\mu_{1} B_{1} P_{C}\left[u_{n-1}-\mu_{2} B_{2} u_{n-1}\right]\right) \|^{2} \\
= & \| P_{C}\left[u_{n}-\mu_{2} B_{2} u_{n}\right]-P_{C}\left[u_{n-1}-\mu_{2} B_{2} u_{n-1}\right] \\
& -\mu_{1}\left(B_{1} P_{C}\left[u_{n}-\mu_{2} B_{2} u_{n}\right]-B_{1} P_{C}\left[u_{n-1}-\mu_{2} B_{2} u_{n-1}\right]\right) \|^{2} \\
\leq & \left\|P_{C}\left[u_{n}-\mu_{2} B_{2} u_{n}\right]-P_{C}\left[u_{n-1}-\mu_{2} B_{2} u_{n-1}\right]\right\|^{2} \\
& -\mu_{1}\left(2 \theta_{1}-\mu_{1}\right)\left\|B_{1} P_{C}\left[u_{n}-\mu_{2} B_{2} u_{n}\right]-B_{1} P_{C}\left[u_{n-1}-\mu_{2} B_{2} u_{n-1}\right]\right\|^{2} \\
\leq & \left\|P_{C}\left[u_{n}-\mu_{2} B_{2} u_{n}\right]-P_{C}\left[u_{n-1}-\mu_{2} B_{2} u_{n-1}\right]\right\|^{2} \\
\leq & \left\|\left(u_{n}-u_{n-1}\right)-\mu_{2}\left(B_{2} u_{n}-B_{2} u_{n-1}\right)\right\|^{2} \\
\leq & \left\|u_{n}-u_{n-1}\right\|^{2}-\mu_{2}\left(2 \theta_{2}-\mu_{2}\right)\left\|B_{2} u_{n}-B_{2} u_{n-1}\right\|^{2} \\
\leq & \left\|u_{n}-u_{n-1}\right\|^{2} .
\end{aligned}
$$

It follows from (3.8) and (3.9) that

$$
\left\|z_{n}-z_{n-1}\right\| \leq\left\|x_{n-1}-x_{n}\right\|+\frac{1}{\mu}\left|r_{n-1}-r_{n}\right|\left(\gamma\|A\| \sigma_{n}+\chi_{n}\right) .
$$

From (3.1) and the above inequality, we get

$$
\begin{aligned}
\left\|y_{n}-y_{n-1}\right\|= & \left\|\beta_{n} S x_{n}+\left(1-\beta_{n}\right) z_{n}-\left(\beta_{n-1} S x_{n-1}+\left(1-\beta_{n-1}\right) z_{n-1}\right)\right\| \\
= & \| \beta_{n}\left(S x_{n}-S x_{n-1}\right)+\left(\beta_{n}-\beta_{n-1}\right) S x_{n-1} \\
& +\left(1-\beta_{n}\right)\left(z_{n}-z_{n-1}\right)+\left(\beta_{n-1}-\beta_{n}\right) z_{n-1} \| \\
\leq & \beta_{n}\left\|x_{n}-x_{n-1}\right\|+\left(1-\beta_{n}\right)\left\|z_{n}-z_{n-1}\right\|+\left|\beta_{n}-\beta_{n-1}\right|\left(\left\|S x_{n-1}\right\|+\left\|z_{n-1}\right\|\right) \\
\leq & \beta_{n}\left\|x_{n}-x_{n-1}\right\|+\left(1-\beta_{n}\right)\left\{\left\|x_{n-1}-x_{n}\right\|+\frac{1}{\mu}\left|r_{n-1}-r_{n}\right|\left(\gamma\|A\| \sigma_{n}+\chi_{n}\right)\right\} \\
& +\left|\beta_{n}-\beta_{n-1}\right|\left(\left\|S x_{n-1}\right\|+\left\|z_{n-1}\right\|\right) \\
\leq & \left\|x_{n}-x_{n-1}\right\|+\frac{1}{\mu}\left|r_{n-1}-r_{n}\right|\left(\gamma\|A\| \sigma_{n}+\chi_{n}\right) \\
& +\left|\beta_{n}-\beta_{n-1}\right|\left(\left\|S x_{n-1}\right\|+\left\|z_{n-1}\right\|\right) .
\end{aligned}
$$

Next, we estimate

$$
\begin{aligned}
\left\|x_{n+1}-x_{n}\right\|= & \left\|P_{C}\left[V_{n}\right]-P_{C}\left[V_{n-1}\right]\right\| \\
\leq & \| \alpha_{n} \rho\left(U\left(x_{n}\right)-U\left(x_{n-1}\right)\right)+\left(\alpha_{n}-\alpha_{n-1}\right) \rho U\left(x_{n-1}\right)+\left(I-\alpha_{n} \mu F\right)\left(T\left(y_{n}\right)\right) \\
& -\left(I-\alpha_{n} \mu F\right) T\left(y_{n-1}\right)+\left(I-\alpha_{n} \mu F\right)\left(T\left(y_{n-1}\right)\right)-\left(I-\alpha_{n-1} \mu F\right)\left(T\left(y_{n-1}\right)\right) \| \\
\leq & \alpha_{n} \rho \tau\left\|x_{n}-x_{n-1}\right\|+\left(1-\alpha_{n} v\right)\left\|y_{n}-y_{n-1}\right\| \\
& +\left|\alpha_{n}-\alpha_{n-1}\right|\left(\left\|\rho U\left(x_{n-1}\right)\right\|+\left\|\mu F\left(T\left(y_{n-1}\right)\right)\right\|\right)
\end{aligned}
$$

where the second inequality follows from Lemma 2.7. From (3.10) and (3.11), we have

$$
\begin{aligned}
\left\|x_{n+1}-x_{n}\right\| \leq & \alpha_{n} \rho \tau\left\|x_{n}-x_{n-1}\right\|+\left(1-\alpha_{n} \nu\right)\left\{\left\|x_{n}-x_{n-1}\right\|+\frac{1}{\mu}\left|r_{n-1}-r_{n}\right|\left(\gamma\|A\| \sigma_{n}+\chi_{n}\right)\right. \\
& \left.+\left|\beta_{n}-\beta_{n-1}\right|\left(\left\|S x_{n-1}\right\|+\left\|z_{n-1}\right\|\right)\right\}
\end{aligned}
$$




$$
\begin{aligned}
& +\left|\alpha_{n}-\alpha_{n-1}\right|\left(\left\|\rho U\left(x_{n-1}\right)\right\|+\left\|\mu F\left(T\left(y_{n-1}\right)\right)\right\|\right) \\
\leq & \left(1-(\nu-\rho \tau) \alpha_{n}\right)\left\|x_{n}-x_{n-1}\right\| \\
& +\frac{1}{\mu}\left|r_{n-1}-r_{n}\right|\left(\gamma\|A\| \sigma_{n}+\chi_{n}\right)+\left|\beta_{n}-\beta_{n-1}\right|\left(\left\|S x_{n-1}\right\|+\left\|z_{n-1}\right\|\right) \\
& +\left|\alpha_{n}-\alpha_{n-1}\right|\left(\left\|\rho U\left(x_{n-1}\right)\right\|+\left\|\mu F\left(T\left(y_{n-1}\right)\right)\right\|\right) \\
\leq & \left(1-(\nu-\rho \tau) \alpha_{n}\right)\left\|x_{n}-x_{n-1}\right\| \\
& +M\left(\frac{1}{\mu}\left|r_{n-1}-r_{n}\right|+\left|\beta_{n}-\beta_{n-1}\right|+\left|\alpha_{n}-\alpha_{n-1}\right|\right) .
\end{aligned}
$$

Here

$$
\begin{aligned}
M= & \max \left\{\sup _{n \geq 1}\left(\gamma\|A\| \sigma_{n}+\chi_{n}\right), \sup _{n \geq 1}\left(\left\|S x_{n-1}\right\|+\left\|z_{n-1}\right\|\right),\right. \\
& \left.\sup _{n \geq 1}\left(\left\|\rho U\left(x_{n-1}\right)\right\|+\left\|\mu F\left(T\left(y_{n-1}\right)\right)\right\|\right)\right\} .
\end{aligned}
$$

It follows by conditions (a)-(d) of Algorithm 3.1 and Lemma 2.8 that

$$
\lim _{n \rightarrow \infty}\left\|x_{n+1}-x_{n}\right\|=0 .
$$

Next, we show that $\lim _{n \rightarrow \infty}\left\|u_{n}-x_{n}\right\|=0$. Since $x^{*} \in S^{*} \cap \Lambda \cap F(T)$ by using (3.2), (3.5), and (3.7), we obtain

$$
\begin{aligned}
\left\|x_{n+1}-x^{*}\right\|^{2}= & \left\langle P_{C}\left[V_{n}\right]-x^{*}, x_{n+1}-x^{*}\right\rangle \\
= & \left\langle P_{C}\left[V_{n}\right]-V_{n}, P_{C}\left[V_{n}\right]-x^{*}\right\rangle+\left\langle V_{n}-x^{*}, x_{n+1}-x^{*}\right\rangle \\
\leq & \left\langle\alpha_{n}\left(\rho U\left(x_{n}\right)-\mu F\left(x^{*}\right)\right)+\left(I-\alpha_{n} \mu F\right)\left(T\left(y_{n}\right)\right)\right. \\
& \left.-\left(I-\alpha_{n} \mu F\right)\left(T\left(x^{*}\right)\right), x_{n+1}-x^{*}\right\rangle \\
= & \left\langle\alpha_{n} \rho\left(U\left(x_{n}\right)-U\left(x^{*}\right)\right), x_{n+1}-x^{*}\right\rangle+\alpha_{n}\left\langle\rho U\left(x^{*}\right)-\mu F\left(x^{*}\right), x_{n+1}-x^{*}\right\rangle \\
& +\left\langle\left(I-\alpha_{n} \mu F\right)\left(T\left(y_{n}\right)\right)-\left(I-\alpha_{n} \mu F\right)\left(T\left(x^{*}\right)\right), x_{n+1}-x^{*}\right\rangle \\
\leq & \alpha_{n} \rho \tau\left\|x_{n}-x^{*}\right\|\left\|x_{n+1}-x^{*}\right\|+\alpha_{n}\left\langle\rho U\left(x^{*}\right)-\mu F\left(x^{*}\right), x_{n+1}-x^{*}\right\rangle \\
& +\left(1-\alpha_{n} v\right)\left\|y_{n}-x^{*}\right\|\left\|x_{n+1}-x^{*}\right\| \\
\leq & \frac{\alpha_{n} \rho \tau}{2}\left(\left\|x_{n}-x^{*}\right\|^{2}+\left\|x_{n+1}-x^{*}\right\|^{2}\right)+\alpha_{n}\left(\rho U\left(x^{*}\right)-\mu F\left(x^{*}\right), x_{n+1}-x^{*}\right\rangle \\
& +\frac{\left(1-\alpha_{n} \nu\right)}{2}\left(\left\|y_{n}-x^{*}\right\|^{2}+\left\|x_{n+1}-x^{*}\right\|^{2}\right) \\
\leq & \frac{\left(1-\alpha_{n}(v-\rho \tau)\right)}{2}\left\|x_{n+1}-x^{*}\right\|^{2}+\frac{\alpha_{n} \rho \tau}{2}\left\|x_{n}-x^{*}\right\|^{2} \\
& +\alpha_{n}\left\langle\rho U\left(x^{*}\right)-\mu F\left(x^{*}\right), x_{n+1}-x^{*}\right\rangle \\
& +\frac{\left(1-\alpha_{n} \nu\right)}{2}\left(\beta_{n}\left\|S x_{n}-x^{*}\right\|^{2}+\left(1-\beta_{n}\right)\left\|z_{n}-x^{*}\right\|^{2}\right) \\
\leq & \frac{\left(1-\alpha_{n}(v-\rho \tau)\right)}{2}\left\|x_{n+1}-x^{*}\right\|^{2}+\frac{\alpha_{n} \rho \tau}{2}\left\|x_{n}-x^{*}\right\|^{2} \\
& +\alpha_{n}\left\langle\rho U\left(x^{*}\right)-\mu F\left(x^{*}\right), x_{n+1}-x^{*}\right\rangle+\frac{\left(1-\alpha_{n} v\right) \beta_{n}}{2}\left\|S x_{n}-x^{*}\right\|^{2} \\
&
\end{aligned}
$$




$$
\begin{aligned}
& +\frac{\left(1-\alpha_{n} v\right)\left(1-\beta_{n}\right)}{2}\left\{\left\|u_{n}-x^{*}\right\|^{2}-\mu_{2}\left(2 \theta_{2}-\mu_{2}\right)\left\|B_{2} u_{n}-B_{2} x^{*}\right\|^{2}\right. \\
& \left.-\mu_{1}\left(2 \theta_{1}-\mu_{1}\right)\left\|B_{1} v_{n}-B_{1} y^{*}\right\|^{2}\right\} \\
\leq & \frac{\left(1-\alpha_{n}(\nu-\rho \tau)\right)}{2}\left\|x_{n+1}-x^{*}\right\|^{2}+\frac{\alpha_{n} \rho \tau}{2}\left\|x_{n}-x^{*}\right\|^{2} \\
& +\alpha_{n}\left\{\rho U\left(x^{*}\right)-\mu F\left(x^{*}\right), x_{n+1}-x^{*}\right\rangle+\frac{\left(1-\alpha_{n} v\right) \beta_{n}}{2}\left\|S x_{n}-x^{*}\right\|^{2} \\
& +\frac{\left(1-\alpha_{n} v\right)\left(1-\beta_{n}\right)}{2}\left\{\left\|x_{n}-x^{*}\right\|^{2}+\gamma(L \gamma-1)\left\|\left(T_{r_{n}}^{F_{2}}-I\right) A x_{n}\right\|^{2}\right. \\
& \left.-\mu_{2}\left(2 \theta_{2}-\mu_{2}\right)\left\|B_{2} u_{n}-B_{2} x^{*}\right\|^{2}-\mu_{1}\left(2 \theta_{1}-\mu_{1}\right)\left\|B_{1} v_{n}-B_{1} y^{*}\right\|^{2}\right\},
\end{aligned}
$$

which implies that

$$
\begin{aligned}
\left\|x_{n+1}-x^{*}\right\|^{2} \leq & \frac{\alpha_{n} \rho \tau}{1+\alpha_{n}(v-\rho \tau)}\left\|x_{n}-x^{*}\right\|^{2} \\
& +\frac{2 \alpha_{n}}{1+\alpha_{n}(v-\rho \tau)}\left\langle\rho U\left(x^{*}\right)-\mu F\left(x^{*}\right), x_{n+1}-x^{*}\right\rangle \\
& +\frac{\left(1-\alpha_{n} v\right) \beta_{n}}{1+\alpha_{n}(v-\rho \tau)}\left\|S x_{n}-x^{*}\right\|^{2} \\
& +\frac{\left(1-\alpha_{n} v\right)\left(1-\beta_{n}\right)}{1+\alpha_{n}(v-\rho \tau)}\left\{\left\|x_{n}-x^{*}\right\|^{2}+\gamma(L \gamma-1)\left\|\left(T_{r_{n}}^{F_{2}}-I\right) A x_{n}\right\|^{2}\right. \\
& \left.-\mu_{2}\left(2 \theta_{2}-\mu_{2}\right)\left\|B_{2} u_{n}-B_{2} x^{*}\right\|^{2}-\mu_{1}\left(2 \theta_{1}-\mu_{1}\right)\left\|B_{1} v_{n}-B_{1} y^{*}\right\|^{2}\right\} \\
\leq & \frac{\alpha_{n} \rho \tau}{1+\alpha_{n}(v-\rho \tau)}\left\|x_{n}-x^{*}\right\|^{2} \\
& +\frac{2 \alpha_{n}}{1+\alpha_{n}(v-\rho \tau)}\left\langle\rho U\left(x^{*}\right)-\mu F\left(x^{*}\right), x_{n+1}-x^{*}\right\rangle \\
& +\left\|x_{n}-x^{*}\right\|^{2}+\frac{\left(1-\alpha_{n} v\right) \beta_{n}}{1+\alpha_{n}(v-\rho \tau)}\left\|S x_{n}-x^{*}\right\|^{2} \\
& -\frac{\left(1-\alpha_{n} v\right)\left(1-\beta_{n}\right)}{1+\alpha_{n}(v-\rho \tau)}\left\{\gamma(1-L \gamma)\left\|\left(T_{r_{n}}^{F_{2}}-I\right) A x_{n}\right\|^{2}\right. \\
& \left.+\mu_{2}\left(2 \theta_{2}-\mu_{2}\right)\left\|B_{2} u_{n}-B_{2} x^{*}\right\|^{2}+\mu_{1}\left(2 \theta_{1}-\mu_{1}\right)\left\|B_{1} v_{n}-B_{1} y^{*}\right\|^{2}\right\} .
\end{aligned}
$$

Then from the above inequality, we get

$$
\begin{aligned}
& \frac{\left(1-\alpha_{n} \nu\right)\left(1-\beta_{n}\right)}{1+\alpha_{n}(v-\rho \tau)}\left\{\gamma(1-L \gamma)\left\|\left(T_{r_{n}}^{F_{2}}-I\right) A x_{n}\right\|^{2}+\mu_{2}\left(2 \theta_{2}-\mu_{2}\right)\left\|B_{2} u_{n}-B_{2} x^{*}\right\|^{2}\right. \\
&\left.\quad+\mu_{1}\left(2 \theta_{1}-\mu_{1}\right)\left\|B_{1} v_{n}-B_{1} y^{*}\right\|^{2}\right\} \\
& \leq \frac{\alpha_{n} \rho \tau}{1+\alpha_{n}(v-\rho \tau)}\left\|x_{n}-x^{*}\right\|^{2}+\frac{2 \alpha_{n}}{1+\alpha_{n}(v-\rho \tau)}\left\langle\rho U\left(x^{*}\right)-\mu F\left(x^{*}\right), x_{n+1}-x^{*}\right\rangle \\
& \quad+\beta_{n}\left\|S x_{n}-x^{*}\right\|^{2}+\left\|x_{n}-x^{*}\right\|^{2}-\left\|x_{n+1}-x^{*}\right\|^{2} \\
& \leq \frac{\alpha_{n} \rho \tau}{1+\alpha_{n}(v-\rho \tau)}\left\|x_{n}-x^{*}\right\|^{2}+\frac{2 \alpha_{n}}{1+\alpha_{n}(v-\rho \tau)}\left\langle\rho U\left(x^{*}\right)-\mu F\left(x^{*}\right), x_{n+1}-x^{*}\right\rangle \\
&+\beta_{n}\left\|S x_{n}-x^{*}\right\|^{2}+\left(\left\|x_{n}-x^{*}\right\|+\left\|x_{n+1}-x^{*}\right\|\right)\left\|x_{n+1}-x_{n}\right\| .
\end{aligned}
$$


Since $\gamma(1-L \gamma)>0,2 \theta_{1}-\mu_{1}>0,2 \theta_{2}-\mu_{2}>0, \lim _{n \rightarrow \infty}\left\|x_{n+1}-x_{n}\right\|=0, \alpha_{n} \rightarrow 0$, and $\beta_{n} \rightarrow 0$, we obtain

$$
\begin{aligned}
& \lim _{n \rightarrow \infty}\left\|B_{2} u_{n}-B_{2} x^{*}\right\|=0, \\
& \lim _{n \rightarrow \infty}\left\|B_{1} v_{n}-B_{1} y^{*}\right\|=0
\end{aligned}
$$

and

$$
\lim _{n \rightarrow \infty}\left\|\left(T_{r_{n}}^{F_{2}}-I\right) A x_{n}\right\|=0
$$

Since $T_{r_{n}}^{F_{1}}$ is firmly nonexpansive, we have

$$
\begin{aligned}
\left\|u_{n}-x^{*}\right\|^{2}= & \left\|T_{r_{n}}^{F_{1}}\left(x_{n}+\gamma A^{*}\left(T_{r_{n}}^{F_{2}}-I\right) A x_{n}\right)-T_{r_{n}}^{F_{1}}\left(x^{*}\right)\right\|^{2} \\
\leq & \left\langle u_{n}-x^{*}, x_{n}+\gamma A^{*}\left(T_{r_{n}}^{F_{2}}-I\right) A x_{n}-x^{*}\right\rangle \\
= & \frac{1}{2}\left\{\left\|u_{n}-x^{*}\right\|^{2}+\left\|x_{n}+\gamma A^{*}\left(T_{r_{n}}^{F_{2}}-I\right) A x_{n}-x^{*}\right\|^{2}\right. \\
& \left.-\left\|u_{n}-x^{*}-\left[x_{n}+\gamma A^{*}\left(T_{r_{n}}^{F_{2}}-I\right) A x_{n}-x^{*}\right]\right\|^{2}\right\} \\
= & \frac{1}{2}\left\{\left\|u_{n}-x^{*}\right\|^{2}+\left\|x_{n}+\gamma A^{*}\left(T_{r_{n}}^{F_{2}}-I\right) A x_{n}-x^{*}\right\|^{2}\right. \\
& \left.-\left\|u_{n}-x_{n}-\gamma A^{*}\left(T_{r_{n}}^{F_{2}}-I\right) A x_{n}\right\|^{2}\right\} \\
\leq & \frac{1}{2}\left\{\left\|u_{n}-x^{*}\right\|^{2}+\left\|x_{n}-x^{*}\right\|^{2}\right. \\
& \left.-\left\|u_{n}-x_{n}-\gamma A^{*}\left(T_{r_{n}}^{F_{2}}-I\right) A x_{n}\right\|^{2}\right\} \\
= & \frac{1}{2}\left\{\left\|u_{n}-x^{*}\right\|^{2}+\left\|x_{n}-x^{*}\right\|^{2}\right. \\
& \left.-\left[\left\|u_{n}-x_{n}\right\|^{2}+\gamma^{2}\left\|A^{*}\left(T_{r_{n}}^{F_{2}}-I\right) A x_{n}\right\|^{2}-2 \gamma\left(u_{n}-x_{n}, A^{*}\left(T_{r_{n}}^{F_{2}}-I\right) A x_{n}\right\rangle\right]\right\} .
\end{aligned}
$$

Hence, we get

$$
\left\|u_{n}-x^{*}\right\|^{2} \leq\left\|x_{n}-x^{*}\right\|^{2}-\left\|u_{n}-x_{n}\right\|^{2}+2 \gamma\left\|A u_{n}-A x_{n}\right\|\left\|\left(T_{r_{n}}^{F_{2}}-I\right) A x_{n}\right\| .
$$

From (3.13), (3.7), and the above inequality, we have

$$
\begin{aligned}
\left\|x_{n+1}-x^{*}\right\|^{2} \leq & \frac{\left(1-\alpha_{n}(v-\rho \tau)\right)}{2}\left\|x_{n+1}-x^{*}\right\|^{2}+\frac{\alpha_{n} \rho \tau}{2}\left\|x_{n}-x^{*}\right\|^{2} \\
& +\alpha_{n}\left\langle\rho U\left(x^{*}\right)-\mu F\left(x^{*}\right), x_{n+1}-x^{*}\right\rangle \\
& +\frac{\left(1-\alpha_{n} \nu\right)}{2}\left(\beta_{n}\left\|S x_{n}-x^{*}\right\|^{2}+\left(1-\beta_{n}\right)\left\|z_{n}-x^{*}\right\|^{2}\right) \\
\leq & \frac{\left(1-\alpha_{n}(v-\rho \tau)\right)}{2}\left\|x_{n+1}-x^{*}\right\|^{2}+\frac{\alpha_{n} \rho \tau}{2}\left\|x_{n}-x^{*}\right\|^{2} \\
& +\alpha_{n}\left\langle\rho U\left(x^{*}\right)-\mu F\left(x^{*}\right), x_{n+1}-x^{*}\right\rangle \\
& +\frac{\left(1-\alpha_{n} \nu\right)}{2}\left(\beta_{n}\left\|S x_{n}-x^{*}\right\|^{2}+\left(1-\beta_{n}\right)\left\|u_{n}-x^{*}\right\|^{2}\right)
\end{aligned}
$$




$$
\begin{aligned}
\leq & \frac{\left(1-\alpha_{n}(\nu-\rho \tau)\right)}{2}\left\|x_{n+1}-x^{*}\right\|^{2}+\frac{\alpha_{n} \rho \tau}{2}\left\|x_{n}-x^{*}\right\|^{2} \\
& +\alpha_{n}\left\{\rho U\left(x^{*}\right)-\mu F\left(x^{*}\right), x_{n+1}-x^{*}\right\rangle \\
& +\frac{\left(1-\alpha_{n} \nu\right)}{2}\left\{\beta_{n}\left\|S x_{n}-x^{*}\right\|^{2}+\left(1-\beta_{n}\right)\left(\left\|x_{n}-x^{*}\right\|^{2}-\left\|u_{n}-x_{n}\right\|^{2}\right.\right. \\
& \left.\left.+2 \gamma\left\|A u_{n}-A x_{n}\right\|\left\|\left(T_{r_{n}}^{F_{2}}-I\right) A x_{n}\right\|\right)\right\},
\end{aligned}
$$

which implies that

$$
\begin{aligned}
\left\|x_{n+1}-x^{*}\right\|^{2} \leq & \frac{\alpha_{n} \rho \tau}{1+\alpha_{n}(v-\rho \tau)}\left\|x_{n}-x^{*}\right\|^{2} \\
& +\frac{2 \alpha_{n}}{1+\alpha_{n}(v-\rho \tau)}\left\{\rho U\left(x^{*}\right)-\mu F\left(x^{*}\right), x_{n+1}-x^{*}\right\rangle \\
& +\frac{\left(1-\alpha_{n} v\right) \beta_{n}}{1+\alpha_{n}(v-\rho \tau)}\left\|S x_{n}-x^{*}\right\|^{2} \\
& +\frac{\left(1-\alpha_{n} v\right)\left(1-\beta_{n}\right)}{1+\alpha_{n}(v-\rho \tau)}\left\{\left\|x_{n}-x^{*}\right\|^{2}-\left\|u_{n}-x_{n}\right\|^{2}\right. \\
& \left.+2 \gamma\left\|A u_{n}-A x_{n}\right\|\left\|\left(T_{r_{n}}^{F_{2}}-I\right) A x_{n}\right\|\right\} \\
\leq & \frac{\alpha_{n} \rho \tau}{1+\alpha_{n}(v-\rho \tau)}\left\|x_{n}-x^{*}\right\|^{2} \\
& +\frac{2 \alpha_{n}}{1+\alpha_{n}(v-\rho \tau)}\left\langle\rho U\left(x^{*}\right)-\mu F\left(x^{*}\right), x_{n+1}-x^{*}\right\rangle \\
& +\frac{\left(1-\alpha_{n} v\right) \beta_{n}}{1+\alpha_{n}(v-\rho \tau)}\left\|S x_{n}-x^{*}\right\|^{2} \\
& +\left\|x_{n}-x^{*}\right\|^{2}+\frac{\left(1-\alpha_{n} \nu\right)\left(1-\beta_{n}\right)}{1+\alpha_{n}(v-\rho \tau)}\left\{-\left\|u_{n}-x_{n}\right\|^{2}\right. \\
& \left.+2 \gamma\left\|A u_{n}-A x_{n}\right\|\left\|\left(T_{r_{n}}^{F_{2}}-I\right) A x_{n}\right\|\right\} .
\end{aligned}
$$

Hence

$$
\begin{aligned}
\frac{\left(1-\alpha_{n} v\right)\left(1-\beta_{n}\right)}{1+\alpha_{n}(v-\rho \tau)}\left\|u_{n}-x_{n}\right\|^{2} \leq & \frac{\alpha_{n} \rho \tau}{1+\alpha_{n}(v-\rho \tau)}\left\|x_{n}-x^{*}\right\|^{2} \\
& +\frac{2 \alpha_{n}}{1+\alpha_{n}(v-\rho \tau)}\left\langle\rho U\left(x^{*}\right)-\mu F\left(x^{*}\right), x_{n+1}-x^{*}\right\rangle \\
& +\frac{\left(1-\alpha_{n} v\right) \beta_{n}}{1+\alpha_{n}(v-\rho \tau)}\left\|S x_{n}-x^{*}\right\|^{2} \\
& +\frac{2\left(1-\alpha_{n} v\right)\left(1-\beta_{n}\right) \gamma}{1+\alpha_{n}(v-\rho \tau)}\left\|A u_{n}-A x_{n}\right\|\left\|\left(T_{r_{n}}^{F_{2}}-I\right) A x_{n}\right\| \\
& +\left\|x_{n}-x^{*}\right\|^{2}-\left\|x_{n+1}-x^{*}\right\|^{2} \\
\leq & \frac{\alpha_{n} \rho \tau}{1+\alpha_{n}(v-\rho \tau)}\left\|x_{n}-x^{*}\right\|^{2} \\
& +\frac{2 \alpha_{n}}{1+\alpha_{n}(v-\rho \tau)}\left\langle\rho U\left(x^{*}\right)-\mu F\left(x^{*}\right), x_{n+1}-x^{*}\right\rangle \\
& +\frac{\left(1-\alpha_{n} v\right) \beta_{n}}{1+\alpha_{n}(v-\rho \tau)}\left\|S x_{n}-x^{*}\right\|^{2}
\end{aligned}
$$




$$
\begin{aligned}
& +\frac{2\left(1-\alpha_{n} v\right)\left(1-\beta_{n}\right) \gamma}{1+\alpha_{n}(v-\rho \tau)}\left\|A u_{n}-A x_{n}\right\|\left\|\left(T_{r_{n}}^{F_{2}}-I\right) A x_{n}\right\| \\
& +\left(\left\|x_{n}-x^{*}\right\|+\left\|x_{n+1}-x^{*}\right\|\right)\left\|x_{n+1}-x_{n}\right\| .
\end{aligned}
$$

Since $\lim _{n \rightarrow \infty}\left\|x_{n+1}-x_{n}\right\|=0, \alpha_{n} \rightarrow 0, \beta_{n} \rightarrow 0$, and $\lim _{n \rightarrow \infty}\left\|\left(T_{r_{n}}^{F_{2}}-I\right) A x_{n}\right\|=0$, we obtain

$$
\lim _{n \rightarrow \infty}\left\|u_{n}-x_{n}\right\|=0
$$

From (2.2), we get

$$
\begin{aligned}
\left\|v_{n}-y^{*}\right\|^{2}= & \left\|P_{C}\left[u_{n}-\mu_{2} B_{2} u_{n}\right]-P_{C}\left[x^{*}-\mu_{2} B_{2} x^{*}\right]\right\|^{2} \\
\leq & \left\langle v_{n}-y^{*},\left(u_{n}-\mu_{2} B_{2} u_{n}\right)-\left(x^{*}-\mu_{2} B_{2} x^{*}\right)\right\rangle \\
= & \frac{1}{2}\left\{\left\|v_{n}-y^{*}\right\|^{2}+\left\|u_{n}-x^{*}-\mu_{2}\left(B_{2} u_{n}-B_{2} x^{*}\right)\right\|^{2}\right. \\
& \left.-\left\|u_{n}-x^{*}-\mu_{2}\left(B_{2} u_{n}-B_{2} x^{*}\right)-\left(v_{n}-y^{*}\right)\right\|^{2}\right\} \\
\leq & \frac{1}{2}\left\{\left\|v_{n}-y^{*}\right\|^{2}+\left\|u_{n}-x^{*}\right\|^{2}-\mu_{2}\left(2 \theta_{2}-\mu_{2}\right)\left\|B_{2} u_{n}-B_{2} x^{*}\right\|^{2}\right. \\
& \left.-\left\|u_{n}-x^{*}-\mu_{2}\left(B_{2} u_{n}-B_{2} x^{*}\right)-\left(v_{n}-y^{*}\right)\right\|^{2}\right\} \\
\leq & \frac{1}{2}\left\{\left\|v_{n}-y^{*}\right\|^{2}+\left\|u_{n}-x^{*}\right\|^{2}-\left\|u_{n}-v_{n}-\mu_{2}\left(B_{2} u_{n}-B_{2} x^{*}\right)-\left(x^{*}-y^{*}\right)\right\|^{2}\right\} \\
= & \frac{1}{2}\left\{\left\|v_{n}-y^{*}\right\|^{2}+\left\|u_{n}-x^{*}\right\|^{2}-\left\|u_{n}-v_{n}-\left(x^{*}-y^{*}\right)\right\|^{2}\right. \\
& \left.+2 \mu_{2}\left\langle u_{n}-v_{n}-\left(x^{*}-y^{*}\right), B_{2} u_{n}-B_{2} x^{*}\right\rangle-\mu_{2}^{2}\left\|B_{2} u_{n}-B_{2} x^{*}\right\|^{2}\right\} \\
\leq & \frac{1}{2}\left\{\left\|v_{n}-y^{*}\right\|^{2}+\left\|u_{n}-x^{*}\right\|^{2}-\left\|u_{n}-v_{n}-\left(x^{*}-y^{*}\right)\right\|^{2}\right. \\
& \left.+2 \mu_{2}\left\|u_{n}-v_{n}-\left(x^{*}-y^{*}\right)\right\|\left\|B_{2} u_{n}-B_{2} x^{*}\right\|\right\} .
\end{aligned}
$$

Hence

$$
\begin{aligned}
\left\|v_{n}-y^{*}\right\|^{2} \leq & \left\|u_{n}-x^{*}\right\|^{2}-\left\|u_{n}-v_{n}-\left(x^{*}-y^{*}\right)\right\|^{2} \\
& +2 \mu_{2}\left\|u_{n}-v_{n}-\left(x^{*}-y^{*}\right)\right\|\left\|B_{2} u_{n}-B_{2} x^{*}\right\| \\
\leq & \left\|x_{n}-x^{*}\right\|^{2}-\left\|u_{n}-x_{n}\right\|^{2}+2 \gamma\left\|A u_{n}-A x_{n}\right\|\left\|\left(T_{r_{n}}^{F_{2}}-I\right) A x_{n}\right\| \\
& \quad-\left\|u_{n}-v_{n}-\left(x^{*}-y^{*}\right)\right\|^{2} \\
& +2 \mu_{2}\left\|u_{n}-v_{n}-\left(x^{*}-y^{*}\right)\right\|\left\|B_{2} u_{n}-B_{2} x^{*}\right\|,
\end{aligned}
$$

where the last inequality follows from (3.15). On the other hand, from (3.1) and (2.2), we obtain

$$
\begin{aligned}
\left\|z_{n}-x^{*}\right\|^{2}= & \left\|P_{C}\left[v_{n}-\mu_{1} B_{1} v_{n}\right]-P_{C}\left[y^{*}-\mu_{1} B_{1} y^{*}\right]\right\|^{2} \\
\leq & \left\langle z_{n}-x^{*},\left(v_{n}-\mu_{1} B_{1} v_{n}\right)-\left(y^{*}-\mu_{1} B_{1} y^{*}\right)\right\rangle \\
= & \frac{1}{2}\left\{\left\|z_{n}-x^{*}\right\|^{2}+\left\|v_{n}-y^{*}-\mu_{1}\left(B_{1} v_{n}-B_{1} y^{*}\right)\right\|^{2}\right. \\
& \left.-\left\|v_{n}-y^{*}-\mu_{1}\left(B_{1} v_{n}-B_{1} y^{*}\right)-\left(z_{n}-x^{*}\right)\right\|^{2}\right\}
\end{aligned}
$$




$$
\begin{aligned}
= & \frac{1}{2}\left\{\left\|z_{n}-x^{*}\right\|^{2}+\left\|v_{n}-y^{*}\right\|^{2}-2 \mu_{1}\left\langle v_{n}-y^{*}, B_{1} v_{n}-B_{1} y^{*}\right\rangle\right. \\
& \left.+\mu_{1}^{2}\left\|B_{1} v_{n}-B_{1} y^{*}\right\|^{2}-\left\|v_{n}-y^{*}-\mu_{1}\left(B_{1} v_{n}-B_{1} y^{*}\right)-\left(z_{n}-x^{*}\right)\right\|^{2}\right\} \\
\leq & \frac{1}{2}\left\{\left\|z_{n}-x^{*}\right\|^{2}+\left\|v_{n}-y^{*}\right\|^{2}-\mu_{1}\left(2 \theta_{1}-\mu_{1}\right)\left\|B_{1} v_{n}-B_{1} y^{*}\right\|^{2}\right. \\
& \left.-\left\|v_{n}-y^{*}-\mu_{1}\left(B_{1} v_{n}-B_{1} y^{*}\right)-\left(z_{n}-x^{*}\right)\right\|^{2}\right\} \\
\leq & \frac{1}{2}\left\{\left\|z_{n}-x^{*}\right\|^{2}+\left\|v_{n}-y^{*}\right\|^{2}\right. \\
& \left.-\left\|v_{n}-z_{n}-\mu_{1}\left(B_{1} v_{n}-B_{1} y^{*}\right)+\left(x^{*}-y^{*}\right)\right\|^{2}\right\} \\
\leq & \frac{1}{2}\left\{\left\|z_{n}-x^{*}\right\|^{2}+\left\|v_{n}-y^{*}\right\|^{2}-\left\|v_{n}-z_{n}+\left(x^{*}-y^{*}\right)\right\|^{2}\right. \\
& \left.+2 \mu_{1}\left\langle v_{n}-z_{n}+\left(x^{*}-y^{*}\right), B_{1} v_{n}-B_{1} y^{*}\right\rangle\right\} \\
\leq & \frac{1}{2}\left\{\left\|z_{n}-x^{*}\right\|^{2}+\left\|v_{n}-y^{*}\right\|^{2}-\left\|v_{n}-z_{n}+\left(x^{*}-y^{*}\right)\right\|^{2}\right. \\
& \left.+2 \mu_{1}\left\|v_{n}-z_{n}+\left(x^{*}-y^{*}\right)\right\|\left\|B_{1} v_{n}-B_{1} y^{*}\right\|\right\} .
\end{aligned}
$$

Hence

$$
\begin{aligned}
\left\|z_{n}-x^{*}\right\|^{2} \leq & \left\|v_{n}-y^{*}\right\|^{2}-\left\|v_{n}-z_{n}+\left(x^{*}-y^{*}\right)\right\|^{2} \\
& +2 \mu_{1}\left\|v_{n}-z_{n}+\left(x^{*}-y^{*}\right)\right\|\left\|B_{1} v_{n}-B_{1} y^{*}\right\| \\
\leq & \left\|x_{n}-x^{*}\right\|^{2}-\left\|u_{n}-x_{n}\right\|^{2}+2 \gamma\left\|A u_{n}-A x_{n}\right\|\left\|\left(T_{r_{n}}^{F_{2}}-I\right) A x_{n}\right\| \\
& -\left\|u_{n}-v_{n}-\left(x^{*}-y^{*}\right)\right\|^{2}+2 \mu_{2}\left\|u_{n}-v_{n}-\left(x^{*}-y^{*}\right)\right\|\left\|B_{2} u_{n}-B_{2} x^{*}\right\| \\
& -\left\|v_{n}-z_{n}+\left(x^{*}-y^{*}\right)\right\|^{2}+2 \mu_{1}\left\|v_{n}-z_{n}+\left(x^{*}-y^{*}\right)\right\|\left\|B_{1} v_{n}-B_{1} y^{*}\right\|,
\end{aligned}
$$

where the last inequality follows from (3.17). From (3.13) and the above inequality, we have

$$
\begin{aligned}
\left\|x_{n+1}-x^{*}\right\|^{2} \leq & \frac{\left(1-\alpha_{n}(v-\rho \tau)\right)}{2}\left\|x_{n+1}-x^{*}\right\|^{2}+\frac{\alpha_{n} \rho \tau}{2}\left\|x_{n}-x^{*}\right\|^{2} \\
& +\alpha_{n}\left\langle\rho U\left(x^{*}\right)-\mu F\left(x^{*}\right), x_{n+1}-x^{*}\right\rangle \\
& +\frac{\left(1-\alpha_{n} v\right)}{2}\left(\beta_{n}\left\|S x_{n}-x^{*}\right\|^{2}+\left(1-\beta_{n}\right)\left\|z_{n}-x^{*}\right\|^{2}\right) \\
\leq & \frac{\left(1-\alpha_{n}(v-\rho \tau)\right)}{2}\left\|x_{n+1}-x^{*}\right\|^{2}+\frac{\alpha_{n} \rho \tau}{2}\left\|x_{n}-x^{*}\right\|^{2} \\
& +\alpha_{n}\left\langle\rho U\left(x^{*}\right)-\mu F\left(x^{*}\right), x_{n+1}-x^{*}\right\rangle \\
& +\frac{\left(1-\alpha_{n} v\right)}{2}\left\{\beta_{n}\left\|S x_{n}-x^{*}\right\|^{2}+\left(1-\beta_{n}\right)\left(\left\|x_{n}-x^{*}\right\|^{2}-\left\|u_{n}-x_{n}\right\|^{2}\right.\right. \\
& \left.+2 \gamma\left\|A u_{n}-A x_{n}\right\|\left\|\left(T_{r_{n}}^{F_{2}}-I\right) A x_{n}\right\|\right) \\
& +\left(1-\beta_{n}\right)\left(-\left\|u_{n}-v_{n}-\left(x^{*}-y^{*}\right)\right\|^{2}\right. \\
& \left.+2 \mu_{2}\left\|u_{n}-v_{n}-\left(x^{*}-y^{*}\right)\right\|\left\|B_{2} u_{n}-B_{2} x^{*}\right\|\right) \\
& +\left(1-\beta_{n}\right)\left(-\left\|v_{n}-z_{n}+\left(x^{*}-y^{*}\right)\right\|^{2}\right. \\
& \left.\left.+2 \mu_{1}\left\|v_{n}-z_{n}+\left(x^{*}-y^{*}\right)\right\|\left\|B_{1} v_{n}-B_{1} y^{*}\right\|\right)\right\}
\end{aligned}
$$


which implies that

$$
\begin{aligned}
& \left\|x_{n+1}-x^{*}\right\|^{2} \leq \frac{\alpha_{n} \rho \tau}{1+\alpha_{n}(v-\rho \tau)}\left\|x_{n}-x^{*}\right\|^{2} \\
& +\frac{2 \alpha_{n}}{1+\alpha_{n}(v-\rho \tau)}\left\langle\rho U\left(x^{*}\right)-\mu F\left(x^{*}\right), x_{n+1}-x^{*}\right\rangle \\
& +\frac{\left(1-\alpha_{n} \nu\right) \beta_{n}}{1+\alpha_{n}(v-\rho \tau)}\left\|S x_{n}-x^{*}\right\|^{2} \\
& +\frac{\left(1-\alpha_{n} \nu\right)\left(1-\beta_{n}\right)}{1+\alpha_{n}(\nu-\rho \tau)}\left\{\left\|x_{n}-x^{*}\right\|^{2}-\left\|u_{n}-x_{n}\right\|^{2}\right. \\
& \left.+2 \gamma\left\|A u_{n}-A x_{n}\right\|\left\|\left(T_{r_{n}}^{F_{2}}-I\right) A x_{n}\right\|\right\} \\
& +\frac{\left(1-\alpha_{n} \nu\right)\left(1-\beta_{n}\right)}{1+\alpha_{n}(\nu-\rho \tau)}\left(-\left\|u_{n}-v_{n}-\left(x^{*}-y^{*}\right)\right\|^{2}\right. \\
& \left.+2 \mu_{2}\left\|u_{n}-v_{n}-\left(x^{*}-y^{*}\right)\right\|\left\|B_{2} u_{n}-B_{2} x^{*}\right\|\right) \\
& +\frac{\left(1-\alpha_{n} \nu\right)\left(1-\beta_{n}\right)}{1+\alpha_{n}(\nu-\rho \tau)}\left(-\left\|v_{n}-z_{n}+\left(x^{*}-y^{*}\right)\right\|^{2}\right. \\
& \left.+2 \mu_{1}\left\|v_{n}-z_{n}+\left(x^{*}-y^{*}\right)\right\|\left\|B_{1} v_{n}-B_{1} y^{*}\right\|\right) \\
& \leq \frac{\alpha_{n} \rho \tau}{1+\alpha_{n}(v-\rho \tau)}\left\|x_{n}-x^{*}\right\|^{2} \\
& +\frac{2 \alpha_{n}}{1+\alpha_{n}(v-\rho \tau)}\left\langle\rho U\left(x^{*}\right)-\mu F\left(x^{*}\right), x_{n+1}-x^{*}\right\rangle \\
& +\frac{\left(1-\alpha_{n} \nu\right) \beta_{n}}{1+\alpha_{n}(\nu-\rho \tau)}\left\|S x_{n}-x^{*}\right\|^{2} \\
& +\left\|x_{n}-x^{*}\right\|^{2}+2 \gamma\left\|A u_{n}-A x_{n}\right\|\left\|\left(T_{r_{n}}^{F_{2}}-I\right) A x_{n}\right\| \\
& +2 \mu_{2}\left\|u_{n}-v_{n}-\left(x^{*}-y^{*}\right)\right\|\left\|B_{2} u_{n}-B_{2} x^{*}\right\| \\
& +2 \mu_{1}\left\|v_{n}-z_{n}+\left(x^{*}-y^{*}\right)\right\|\left\|B_{1} v_{n}-B_{1} y^{*}\right\| \\
& -\frac{\left(1-\alpha_{n} \nu\right)\left(1-\beta_{n}\right)}{1+\alpha_{n}(\nu-\rho \tau)}\left(\left\|u_{n}-x_{n}\right\|^{2}+\left\|u_{n}-v_{n}-\left(x^{*}-y^{*}\right)\right\|^{2}\right. \\
& \left.+\left\|v_{n}-z_{n}+\left(x^{*}-y^{*}\right)\right\|^{2}\right) \text {. }
\end{aligned}
$$

Hence

$$
\begin{aligned}
& \frac{\left(1-\alpha_{n} v\right)\left(1-\beta_{n}\right)}{1+\alpha_{n}(v-\rho \tau)}\left(\left\|u_{n}-x_{n}\right\|^{2}+\left\|u_{n}-v_{n}-\left(x^{*}-y^{*}\right)\right\|^{2}+\left\|v_{n}-z_{n}+\left(x^{*}-y^{*}\right)\right\|^{2}\right) \\
& \leq \frac{\alpha_{n} \rho \tau}{1+\alpha_{n}(v-\rho \tau)}\left\|x_{n}-x^{*}\right\|^{2} \\
& \quad+\frac{2 \alpha_{n}}{1+\alpha_{n}(v-\rho \tau)}\left\langle\rho U\left(x^{*}\right)-\mu F\left(x^{*}\right), x_{n+1}-x^{*}\right\rangle+\frac{\left(1-\alpha_{n} v\right) \beta_{n}}{1+\alpha_{n}(v-\rho \tau)}\left\|S x_{n}-x^{*}\right\|^{2} \\
& \quad+\left\|x_{n}-x^{*}\right\|^{2}-\left\|x_{n+1}-x^{*}\right\|^{2}+2 \gamma\left\|A u_{n}-A x_{n}\right\|\left\|\left(T_{r_{n}}^{F_{2}}-I\right) A x_{n}\right\| \\
& \quad+2 \mu_{2}\left\|u_{n}-v_{n}-\left(x^{*}-y^{*}\right)\right\|\left\|B_{2} u_{n}-B_{2} x^{*}\right\| \\
& \quad+2 \mu_{1}\left\|v_{n}-z_{n}+\left(x^{*}-y^{*}\right)\right\|\left\|B_{1} v_{n}-B_{1} y^{*}\right\| \\
& =\frac{\alpha_{n} \rho \tau}{1+\alpha_{n}(v-\rho \tau)}\left\|x_{n}-x^{*}\right\|^{2}
\end{aligned}
$$




$$
\begin{aligned}
& +\frac{2 \alpha_{n}}{1+\alpha_{n}(v-\rho \tau)}\left\langle\rho U\left(x^{*}\right)-\mu F\left(x^{*}\right), x_{n+1}-x^{*}\right\rangle+\frac{\left(1-\alpha_{n} v\right) \beta_{n}}{1+\alpha_{n}(v-\rho \tau)}\left\|S x_{n}-x^{*}\right\|^{2} \\
& +\left(\left\|x_{n}-x^{*}\right\|+\left\|x_{n+1}-x^{*}\right\|\right)\left\|x_{n+1}-x_{n}\right\|+2 \gamma\left\|A u_{n}-A x_{n}\right\|\left\|\left(T_{r_{n}}^{F_{2}}-I\right) A x_{n}\right\| \\
& +2 \mu_{2}\left\|u_{n}-v_{n}-\left(x^{*}-y^{*}\right)\right\|\left\|B_{2} u_{n}-B_{2} x^{*}\right\| \\
& +2 \mu_{1}\left\|v_{n}-z_{n}+\left(x^{*}-y^{*}\right)\right\|\left\|B_{1} v_{n}-B_{1} y^{*}\right\| .
\end{aligned}
$$

Since $\lim _{n \rightarrow \infty}\left\|x_{n+1}-x_{n}\right\|=0, \alpha_{n} \rightarrow 0, \beta_{n} \rightarrow 0$, and $\lim _{n \rightarrow \infty}\left\|\left(T_{r_{n}}^{F_{2}}-I\right) A x_{n}\right\|=0$, $\lim _{n \rightarrow \infty}\left\|B_{2} u_{n}-B_{2} x^{*}\right\|=0, \lim _{n \rightarrow \infty}\left\|B_{1} v_{n}-B_{1} y^{*}\right\|=0$, we obtain

$$
\lim _{n \rightarrow \infty}\left\|u_{n}-v_{n}-\left(x^{*}-y^{*}\right)\right\|=0 \quad \text { and } \quad \lim _{n \rightarrow \infty}\left\|v_{n}-z_{n}+\left(x^{*}-y^{*}\right)\right\|=0 .
$$

Since

$$
\left\|u_{n}-z_{n}\right\| \leq\left\|u_{n}-v_{n}-\left(x^{*}-y^{*}\right)\right\|+\left\|v_{n}-z_{n}+\left(x^{*}-y^{*}\right)\right\|
$$

we get

$$
\lim _{n \rightarrow \infty}\left\|u_{n}-z_{n}\right\|=0
$$

It follows from (3.16) and (3.18) that

$$
\lim _{n \rightarrow \infty}\left\|x_{n}-z_{n}\right\|=0
$$

Since $T\left(x_{n}\right) \in C$, we have

$$
\begin{aligned}
\left\|x_{n}-T\left(x_{n}\right)\right\| \leq & \left\|x_{n}-x_{n+1}\right\|+\left\|x_{n+1}-T\left(x_{n}\right)\right\| \\
& =\left\|x_{n}-x_{n+1}\right\|+\left\|P_{C}\left[V_{n}\right]-P_{C}\left[T\left(x_{n}\right)\right]\right\| \\
\leq & \left\|x_{n}-x_{n+1}\right\|+\left\|\alpha_{n}\left(\rho U\left(x_{n}\right)-\mu F\left(T\left(y_{n}\right)\right)\right)+T\left(y_{n}\right)-T\left(x_{n}\right)\right\| \\
\leq & \left\|x_{n}-x_{n+1}\right\|+\alpha_{n}\left\|\rho U\left(x_{n}\right)-\mu F\left(T\left(y_{n}\right)\right)\right\|+\left\|y_{n}-x_{n}\right\| \\
\leq & \left\|x_{n}-x_{n+1}\right\|+\alpha_{n}\left\|\rho U\left(x_{n}\right)-\mu F\left(T\left(y_{n}\right)\right)\right\|+\left\|\beta_{n} S x_{n}+\left(1-\beta_{n}\right) z_{n}-x_{n}\right\| \\
\leq & \left\|x_{n}-x_{n+1}\right\|+\alpha_{n}\left\|\rho U\left(x_{n}\right)-\mu F\left(T\left(y_{n}\right)\right)\right\| \\
& +\beta_{n}\left\|S x_{n}-x_{n}\right\|+\left(1-\beta_{n}\right)\left\|z_{n}-x_{n}\right\| .
\end{aligned}
$$

Since $\lim _{n \rightarrow \infty}\left\|x_{n+1}-x_{n}\right\|=0, \alpha_{n} \rightarrow 0, \beta_{n} \rightarrow 0$, and $\left\|\rho U\left(x_{n}\right)-\mu F\left(T\left(y_{n}\right)\right)\right\|$ and $\left\|S x_{n}-x_{n}\right\|$ are bounded and $\lim _{n \rightarrow \infty}\left\|x_{n}-z_{n}\right\|=0$, we obtain

$$
\lim _{n \rightarrow \infty}\left\|x_{n}-T\left(x_{n}\right)\right\|=0 .
$$

Since $\left\{x_{n}\right\}$ is bounded, without loss of generality we can assume that $x_{n} \rightarrow x^{*} \in C$. It follows from Lemma 2.5 that $x^{*} \in F(T)$. Therefore $w_{w}\left(x_{n}\right) \subset F(T)$.

Theorem 3.1 The sequence $\left\{x_{n}\right\}$ generated by Algorithm 3.1 converges strongly to $z$, which is the unique solution of the variational inequality

$$
\langle\rho U(z)-\mu F(z), x-z\rangle \leq 0, \quad \forall x \in S^{*} \cap \Lambda \cap F(T) .
$$


Proof Since $\left\{x_{n}\right\}$ is bounded $x_{n} \rightarrow w$ and from Lemma 3.2, we have $w \in F(T)$. Next, we show that $w \in E P\left(F_{1}\right)$. Since $u_{n}=T_{r_{n}}^{F_{1}}\left(x_{n}+\gamma A^{*}\left(T_{r_{n}}^{F_{2}}-I\right) A x_{n}\right)$, we have

$$
F_{1}\left(u_{n}, y\right)+\frac{1}{r_{n}}\left\langle y-u_{n}, u_{n}-x_{n}\right\rangle-\frac{1}{r_{n}}\left\langle y-u_{n}, \gamma A^{*}\left(T_{r_{n}}^{F_{2}}-I\right) A x_{n}\right\rangle \geq 0, \quad \forall y \in C .
$$

It follows from monotonicity of $F_{1}$ that

$$
-\frac{1}{r_{n}}\left\langle y-u_{n}, \gamma A^{*}\left(T_{r_{n}}^{F_{2}}-I\right) A x_{n}\right\rangle+\frac{1}{r_{n}}\left\langle y-u_{n}, u_{n}-x_{n}\right\rangle \geq F_{1}\left(y, u_{n}\right), \quad \forall y \in C
$$

and

$$
-\frac{1}{r_{n_{k}}}\left\langle y-u_{n_{k}}, \gamma A^{*}\left(T_{r_{n_{k}}}^{F_{2}}-I\right) A x_{n_{k}}\right\rangle+\left\langle y-u_{n_{k}}, \frac{u_{n_{k}}-x_{n_{k}}}{r_{n_{k}}}\right\rangle \geq F_{1}\left(y, u_{n_{k}}\right), \quad \forall y \in C .
$$

Since $\lim _{n \rightarrow \infty}\left\|u_{n}-x_{n}\right\|=0, \lim _{n \rightarrow \infty}\left\|\left(T_{r_{n}}^{F_{2}}-I\right) A x_{n}\right\|=0$, and $x_{n} \rightarrow w$, it is easy to observe that $u_{n_{k}} \rightarrow w$. It follows by Assumption 2.1(iv) that $F_{1}(y, w) \leq 0, \forall y \in C$.

For any $0<t \leq 1$ and $y \in C$, let $y_{t}=t y+(1-t) w$, and we have $y_{t} \in C$. Then, from Assumption 2.1(i) and (iv), we have

$$
\begin{aligned}
0=F_{1}\left(y_{t}, y_{t}\right) & \leq t F_{1}\left(y_{t}, y\right)+(1-t) F_{1}\left(y_{t}, w\right) \\
& \leq t F_{1}\left(y_{t}, y\right) .
\end{aligned}
$$

Therefore $F_{1}\left(y_{t}, y\right) \geq 0$. From Assumption 2.1(iii), we have $F_{1}(w, y) \geq 0$, which implies that $w \in E P\left(F_{1}\right)$.

Next, we show that $A w \in E P\left(F_{2}\right)$. Since $\left\{x_{n}\right\}$ is bounded and $x_{n} \rightarrow w$, there exists a subsequence $\left\{x_{n_{k}}\right\}$ of $\left\{x_{n}\right\}$ such that $x_{n_{k}} \rightarrow w$, and since $A$ is a bounded linear operator $A x_{n_{k}} \rightarrow A w$. Now we set $v_{n_{k}}=A x_{n_{k}}-T_{r_{n_{k}}}^{F_{2}} A x_{n_{k}}$. It follows from (3.14) that $\lim _{k \rightarrow \infty} v_{n_{k}}=0$ and $A x_{n_{k}}-v_{n_{k}}=T_{r_{n_{k}}}^{F_{2}} A x_{n_{k}}$. Therefore from the definition of $T_{r_{n_{k}}}^{F_{2}}$, we have

$$
F_{2}\left(A x_{n_{k}}-v_{n_{k}}, y\right)+\frac{1}{r_{n_{k}}}\left\langle y-\left(A x_{n_{k}}-v_{n_{k}}\right),\left(A x_{n_{k}}-v_{n_{k}}\right)-A x_{n_{k}}\right\rangle \geq 0, \quad \forall y \in C .
$$

Since $F_{2}$ is upper semicontinuous in the first argument, taking lim sup in the above inequality as $k \rightarrow \infty$ and using Assumption 2.1(iv), we obtain

$$
F_{2}(A w, y) \geq 0, \quad \forall y \in C,
$$

which implies that $A w \in E P\left(F_{2}\right)$ and hence $w \in \Lambda$.

Next, we show that $w \in S^{*}$. Since $\lim _{n \rightarrow \infty}\left\|x_{n}-z_{n}\right\|=0$ and there exists a subsequence $\left\{x_{n_{k}}\right\}$ of $\left\{x_{n}\right\}$ such that $x_{n_{k}} \rightarrow w$, it is easy to observe that $z_{n_{k}} \rightarrow w$. For any $x, y \in C$, using (2.5), we have

$$
\begin{aligned}
\|Q(x)-Q(y)\|^{2}= & \| P_{C}\left[P_{C}\left[x-\mu_{2} B_{2} x\right]-\mu_{1} B_{1} P_{C}\left[x-\mu_{2} B_{2} x\right]\right] \\
& -P_{C}\left[P_{C}\left[y-\mu_{2} B_{2} y\right]-\mu_{1} B_{1} P_{C}\left[y-\mu_{2} B_{2} y\right]\right] \|^{2} \\
\leq & \|\left(P_{C}\left[x-\mu_{2} B_{2} x\right]-P_{C}\left[y-\mu_{2} B_{2} y\right]\right)
\end{aligned}
$$




$$
\begin{aligned}
& -\mu_{1}\left(B_{1} P_{C}\left[x-\mu_{2} B_{2} x\right]-B_{1} P_{C}\left[y-\mu_{2} B_{2} y\right]\right) \|^{2} \\
\leq & \left\|P_{C}\left[x-\mu_{2} B_{2} x\right]-P_{C}\left[y-\mu_{2} B_{2} y\right]\right\|^{2} \\
& -\mu_{1}\left(2 \theta_{1}-\mu_{1}\right)\left\|B_{1} P_{C}\left[x-\mu_{2} B_{2} x\right]-B_{1} P_{C}\left[y-\mu_{2} B_{2} y\right]\right\|^{2} \\
\leq & \left\|P_{C}\left[x-\mu_{2} B_{2} x\right]-P_{C}\left[y-\mu_{2} B_{2} y\right]\right\|^{2} \\
\leq & \left\|\left(x-\mu_{2} B_{2} x\right)-\left(y-\mu_{2} B_{2} y\right)\right\|^{2} \\
\leq & \|x-y\|^{2}-\mu_{2}\left(2 \theta_{2}-\mu_{2}\right)\left\|B_{2} x-B_{2} y\right\|^{2} \\
\leq & \|x-y\|^{2} .
\end{aligned}
$$

This implies that $Q: C \rightarrow C$ is nonexpansive. On the other hand

$$
\begin{aligned}
\left\|z_{n}-Q\left(z_{n}\right)\right\|^{2} & =\left\|P_{C}\left[P_{C}\left[u_{n}-\mu_{2} B_{2} u_{n}\right]-\mu_{1} B_{1} P_{C}\left[u_{n}-\mu_{2} B_{2} u_{n}\right]\right]-Q\left(z_{n}\right)\right\|^{2} \\
& =\left\|Q\left(u_{n}\right)-Q\left(z_{n}\right)\right\|^{2} \\
& \leq\left\|u_{n}-z_{n}\right\|^{2} .
\end{aligned}
$$

Since $\lim _{n \rightarrow \infty}\left\|u_{n}-z_{n}\right\|=0$ (see (3.18)), we have $\lim _{n \rightarrow \infty}\left\|z_{n}-Q\left(z_{n}\right)\right\|=0$. It follows from Lemma 2.5 that $w=Q(w)$, which implies from Lemma 2.2 that $w \in S^{*}$.

Thus we have

$$
w \in S^{*} \cap \Lambda \cap F(T) .
$$

Observe that the constants satisfy $0 \leq \rho \tau<\nu$ and

$$
\begin{aligned}
k \geq \eta & \Longleftrightarrow k^{2} \geq \eta^{2} \\
& \Longleftrightarrow 1-2 \mu \eta+\mu^{2} k^{2} \geq 1-2 \mu \eta+\mu^{2} \eta^{2} \\
& \Longleftrightarrow \sqrt{1-\mu\left(2 \eta-\mu k^{2}\right)} \geq 1-\mu \eta \\
& \Longleftrightarrow \mu \eta \geq 1-\sqrt{1-\mu\left(2 \eta-\mu k^{2}\right)} \\
& \Longleftrightarrow \mu \eta \geq v ;
\end{aligned}
$$

therefore, from Lemma 2.6, the operator $\mu F-\rho U$ is $\mu \eta-\rho \tau$ strongly monotone, and we get the uniqueness of the solution of the variational inequality (3.20) and denote it by $z \in S^{*} \cap \Lambda \cap F(T)$.

Next, we claim that $\lim _{\sup _{n \rightarrow \infty}}\left\langle\rho U(z)-\mu F(z), x_{n}-z\right\rangle \leq 0$. Since $\left\{x_{n}\right\}$ is bounded, there exists a subsequence $\left\{x_{n_{k}}\right\}$ of $\left\{x_{n}\right\}$ such that

$$
\begin{aligned}
\limsup _{n \rightarrow \infty}\left\langle\rho U(z)-\mu F(z), x_{n}-z\right\rangle & =\limsup _{k \rightarrow \infty}\left\langle\rho U(z)-\mu F(z), x_{n_{k}}-z\right\rangle \\
& =\langle\rho U(z)-\mu F(z), w-z\rangle \leq 0 .
\end{aligned}
$$

Next, we show that $x_{n} \rightarrow z$. We have

$$
\begin{aligned}
\left\|x_{n+1}-z\right\|^{2} & =\left\langle P_{C}\left[V_{n}\right]-z, x_{n+1}-z\right\rangle \\
& =\left\langle P_{C}\left[V_{n}\right]-V_{n}, P_{C}\left[V_{n}\right]-z\right\rangle+\left\langle V_{n}-z, x_{n+1}-z\right\rangle
\end{aligned}
$$




$$
\begin{aligned}
\leq & \left\langle\alpha_{n}\left(\rho U\left(x_{n}\right)-\mu F(z)\right)+\left(I-\alpha_{n} \mu F\right)\left(T\left(y_{n}\right)\right)-\left(I-\alpha_{n} \mu F\right)(T(z)), x_{n+1}-z\right\rangle \\
\leq & \left\langle\alpha_{n} \rho\left(U\left(x_{n}\right)-U(z)\right), x_{n+1}-z\right\rangle+\alpha_{n}\left\langle\rho U(z)-\mu F(z), x_{n+1}-z\right\rangle \\
& +\left\langle\left(I-\alpha_{n} \mu F\right)\left(T\left(y_{n}\right)\right)-\left(I-\alpha_{n} \mu F\right)(T(z)), x_{n+1}-z\right\rangle \\
\leq & \alpha_{n} \rho \tau\left\|x_{n}-z\right\|\left\|x_{n+1}-z\right\|+\alpha_{n}\left\langle\rho U(z)-\mu F(z), x_{n+1}-z\right\rangle \\
& +\left(1-\alpha_{n} v\right)\left\|y_{n}-z\right\|\left\|x_{n+1}-z\right\| \\
\leq & \alpha_{n} \rho \tau\left\|x_{n}-z\right\|\left\|x_{n+1}-z\right\|+\alpha_{n}\left\langle\rho U(z)-\mu F(z), x_{n+1}-z\right\rangle \\
& +\left(1-\alpha_{n} v\right)\left\{\beta_{n}\left\|S x_{n}-S z\right\|+\beta_{n}\|S z-z\|+\left(1-\beta_{n}\right)\left\|z_{n}-z\right\|\right\}\left\|x_{n+1}-z\right\| \\
\leq & \alpha_{n} \rho \tau\left\|x_{n}-z\right\|\left\|x_{n+1}-z\right\|+\alpha_{n}\left\langle\rho U(z)-\mu F(z), x_{n+1}-z\right\rangle \\
& +\left(1-\alpha_{n} \nu\right)\left\{\beta_{n}\left\|x_{n}-z\right\|+\beta_{n}\|S z-z\|+\left(1-\beta_{n}\right)\left\|x_{n}-z\right\|\right\}\left\|x_{n+1}-z\right\| \\
= & \left(1-\alpha_{n}(\nu-\rho \tau)\right)\left\|x_{n}-z\right\|\left\|x_{n+1}-z\right\|+\alpha_{n}\left\langle\rho U(z)-\mu F(z), x_{n+1}-z\right\rangle \\
& +\left(1-\alpha_{n} v\right) \beta_{n}\|S z-z\|\left\|x_{n+1}-z\right\| \\
\leq & \frac{1-\alpha_{n}(\nu-\rho \tau)}{2}\left(\left\|x_{n}-z\right\|^{2}+\left\|x_{n+1}-z\right\|^{2}\right)+\alpha_{n}\left\langle\rho U(z)-\mu F(z), x_{n+1}-z\right\rangle \\
& +\left(1-\alpha_{n} \nu\right) \beta_{n}\|S z-z\|\left\|x_{n+1}-z\right\|,
\end{aligned}
$$

which implies that

$$
\begin{aligned}
\left\|x_{n+1}-z\right\|^{2} \leq & \frac{1-\alpha_{n}(v-\rho \tau)}{1+\alpha_{n}(v-\rho \tau)}\left\|x_{n}-z\right\|^{2}+\frac{2 \alpha_{n}}{1+\alpha_{n}(v-\rho \tau)}\left\langle\rho U(z)-\mu F(z), x_{n+1}-z\right\rangle \\
& +\frac{2\left(1-\alpha_{n} \nu\right) \beta_{n}}{1+\alpha_{n}(v-\rho \tau)}\|S z-z\|\left\|x_{n+1}-z\right\| \\
\leq & \left(1-\alpha_{n}(v-\rho \tau)\right)\left\|x_{n}-z\right\|^{2} \\
& +\frac{2 \alpha_{n}(v-\rho \tau)}{1+\alpha_{n}(v-\rho \tau)}\left\{\frac{1}{v-\rho \tau}\left\langle\rho U(z)-\mu F(z), x_{n+1}-z\right\rangle\right. \\
& \left.+\frac{\left(1-\alpha_{n} \nu\right) \beta_{n}}{\alpha_{n}(v-\rho \tau)}\|S z-z\|\left\|x_{n+1}-z\right\|\right\} .
\end{aligned}
$$

Let $\gamma_{n}=\alpha_{n}(v-\rho \tau)$ and $\delta_{n}=\frac{2 \alpha_{n}(v-\rho \tau)}{1+\alpha_{n}(v-\rho \tau)}\left\{\frac{1}{v-\rho \tau}\left\langle\rho U(z)-\mu F(z), x_{n+1}-z\right\rangle+\frac{\left(1-\alpha_{n} v\right) \beta_{n}}{\alpha_{n}(v-\rho \tau)} \| S z-\right.$ $\left.z\|\| x_{n+1}-z \|\right\}$.

We have

$$
\sum_{n=1}^{\infty} \alpha_{n}=\infty
$$

and

$$
\limsup _{n \rightarrow \infty}\left\{\frac{1}{v-\rho \tau}\left\langle\rho U(z)-\mu F(z), x_{n+1}-z\right\rangle+\frac{\left(1-\alpha_{n} \nu\right) \beta_{n}}{\alpha_{n}(v-\rho \tau)}\|S z-z\|\left\|x_{n+1}-z\right\|\right\} \leq 0
$$

It follows that

$$
\sum_{n=1}^{\infty} \gamma_{n}=\infty \quad \text { and } \quad \limsup _{n \rightarrow \infty} \frac{\delta_{n}}{\gamma_{n}} \leq 0
$$


Thus all the conditions of Lemma 2.8 are satisfied. Hence we deduce that $x_{n} \rightarrow z$. This completes the proof.

Remark 3.2 In hierarchical fixed-point problem (1.11), if $S=I-(\rho U-\mu F)$, then we can get the variational inequality (3.20). In (3.20), if $U=0$ then we get the variational inequality $\langle F(z), x-z\rangle \geq 0, \forall x \in S^{*} \cap \Lambda \cap F(T)$, which just is the variational inequality studied by Suzuki [39] extending the common set of solutions of a system of variational inequalities, a split equilibrium problem, and a hierarchical fixed-point problem.

\section{Conclusions}

In this paper, we suggest and analyze an iterative method for finding the approximate element of the common set of solutions of (1.1), (1.9)-(1.10), and (1.11) in real Hilbert space, which can be viewed as a refinement and improvement of some existing methods for solving a system of variational inequality problem, a split equilibrium problem, and a hierarchical fixed-point problem. Some existing methods (e.g. [20, 26, 34, 40, 45]) can be viewed as special cases of Algorithm 3.1. Therefore, the new algorithm is expected to be widely applicable.

\section{Competing interests}

The author declares that he has no competing interests.

Received: 2 September 2013 Accepted: 30 December 2013 Published: 23 Jan 2014

\section{References}

1. Ceng, LC, Wang, CY, Yao, JC: Strong convergence theorems by a relaxed extragradient method for a general system of variational inequalities. Math. Methods Oper. Res. 67, 375-390 (2008)

2. Ceng, LC, Al-Mezel, SA Anasri, QH: Implicit and explicit iterative methods for systems of variational inequalities and zeros of accretive operators. Abstr. Appl. Anal. 2013, Article ID 631382 (2013)

3. Ansari, QH, Yao, JC: Systems of generalized variational inequalities and their applications. Appl. Anal. 76, 203-217 (2000)

4. Aubin, JP: Mathematical Methods of Game and Economic Theory. North-Holland, Amsterdam (1979)

5. Facchinei, F, Pang, JS: Finite-Dimensional Variational Inequalities and Complementarity Problems, vol. I. Springer, New York (2003)

6. Facchinei, F, Pang, JS: Finite-Dimensional Variational Inequalities and Complementarity Problems, vol. II. Springer, New York (2003)

7. Ansari, QH, Lalitha, CS, Mehta, M: Generalized Convexity, Nonsmooth Variational Inequalities and Nonsmooth Optimization. CRC Press, Boca Raton (2013)

8. Ceng, LC, Anasri, QH, Yao, JC: Iterative methods for triple hierarchical variational inequalities in Hilbert spaces. J. Optim. Theory Appl. 151, 489-512 (2011)

9. Marino, G, Muglia, L, Yao, Y: Viscosity methods for common solutions of equilibrium and variational inequality problems via multi-step iterative algorithms and common fixed points. Nonlinear Anal. 75, 1787-1798 (2012)

10. Latif, A, Ceng, LC, Ansari, QH: Multi-step hybrid viscosity method for systems of variational inequalities defined over sets of solutions of equilibrium problem and fixed point problems. Fixed Point Theory Appl. 2012, 186 (2012)

11. Verma, RU: Projection methods, algorithms, and a new system of nonlinear variational inequalities. Comput. Math. Appl. 41, 1025-1031 (2001)

12. Verma, RU: General convergence analysis for two-step projection methods and applications to variational problems. Appl. Math. Lett. 18, 1286-1292 (2005)

13. Acedo, GL, Xu, HK: Iterative methods for strictly pseudo-contractions in Hilbert space. Nonlinear Anal. 67, 2258-2271 (2007)

14. Blum, E, Oettli, W: From optimization and variational inequalities to equilibrium problems. Math. Stud. 63, 123-145 (1994)

15. Bnouhachem, A, Noor, MA: An iterative method for approximating the common solutions of a variational inequality, a mixed equilibrium problem and a hierarchical fixed point problem. J. Inequal. Appl. 2013, 490 (2013)

16. Bnouhachem, A: Algorithms of common solutions for a variational inequality, a split equilibrium problem and a hierarchical fixed point problem. Fixed Point Theory Appl. 2013, 278 (2013)

17. Bnouhachem, A: Strong convergence algorithm for split equilibrium problems and hierarchical fixed point problems Sci. World J. (in press)

18. Byrne, C, Censor, Y, Gibali, A, Reich, S: Weak and strong convergence of algorithms for the split common null point problem. arXiv:1108.5953

19. Censor, Y, Elfving, T: A multiprojection algorithm using Bregman projections in a product space. Numer. Algorithms 8 , 221-239 (1994) 
20. Ceng, LC, Anasri, QH, Yao, JC: Some iterative methods for finding fixed points and for solving constrained convex minimization problems. Nonlinear Anal. 74, 5286-5302 (2011)

21. Ceng, LC, Anasri, QH, Yao, JC: Mann type iterative methods for finding a common solution of split feasibility and fixed point problems. Positivity 16(3), 471-495 (2012)

22. Ceng, LC, Anasri, QH, Yao, JC: An extragradient method for split feasibility and fixed point problems. Comput. Math. Appl. 64, 633-642 (2012)

23. Censor, Y, Gibali, A, Reich, S: Algorithms for the split variational inequality problem. Numer. Algorithms 59(2), 301-323 (2012)

24. Chang, SS, Lee, HWJ, Chan, CK: Generalized system for relaxed cocoercive variational inequalities in Hilbert spaces. Appl. Math. Lett. 20, 329-334 (2007)

25. Chang, SS, Lee, HWJ, Chan, CK: A new method for solving equilibrium problem fixed point problem and variational inequality problem with application to optimization. Nonlinear Anal. 70, 3307-3319 (2009)

26. Cianciaruso, F, Marino, G, Muglia, L, Yao, Y: On a two-steps algorithm for hierarchical fixed point problems and variational inequalities. J. Inequal. Appl. 2009, Article ID 208692 (2009)

27. Cianciaruso, F, Marino, G, Muglia, L, Yao, Y: A hybrid projection algorithm for finding solutions of mixed equilibrium problem and variational inequality problem. Fixed Point Theory Appl. 2010, Article ID 383740 (2010)

28. Combettes, PL, Hirstoaga, SA: Equilibrium programming using proximal like algorithms. Math. Program. 78, 29-41 (1997)

29. Crombez, G: A geometrical look at iterative methods for operators with fixed points. Numer. Funct. Anal. Optim. 26 157-175 (2005)

30. Crombez, G: A hierarchical presentation of operators with fixed points on Hilbert spaces. Numer. Funct. Anal. Optim. 27, 259-277 (2006)

31. Geobel, K, Kirk, WA: Topics in Metric Fixed Point Theory. Cambridge Stud. Adv. Math., vol. 28. Cambridge University Press, Cambridge (1990)

32. Gu, G, Wang, S, Cho, YJ: Strong convergence algorithms for hierarchical fixed points problems and variational inequalities. J. Appl. Math. 2011, Article ID 164978 (2011)

33. Korpelevic, GM: An extragradient method for finding saddle points and for other problems. Èkon. Mat. Metody 12(4), 747-756 (1976)

34. Mainge, PE, Moudafi, A: Strong convergence of an iterative method for hierarchical fixed-point problems. Pac J. Optim. 3(3), 529-538 (2007)

35. Marino, G, Xu, HK: Explicit hierarchical fixed point approach to variational inequalities. J. Optim. Theory Appl. 149(1), 61-78 (2011)

36. Moudafi, A: Split monotone variational inclusions. J. Optim. Theory Appl. 50, 275-283 (2011)

37. Moudafi, A: Krasnoselski-Mann iteration for hierarchical fixed-point problems. Inverse Probl. 23(4), 1635-1640 (2007)

38. Qin, X, Shang, M, Su, Y: A general iterative method for equilibrium problem and fixed point problem in Hilbert spaces. Nonlinear Anal. 69, 3897-3909 (2008)

39. Suzuki, N: Moudafi's viscosity approximations with Meir-Keeler contractions. J. Math. Anal. Appl. 325, 342-352 (2007)

40. Tian, M: A general iterative algorithm for nonexpansive mappings in Hilbert spaces. Nonlinear Anal. 73, 689-694 (2010)

41. Verma, RU: Generalized system for relaxed cocoercive variational inequalities and projection methods. J. Optim. Theory Appl. 121(1), 203-210 (2004)

42. Wang, $Y, X u, W$ : Strong convergence of a modified iterative algorithm for hierarchical fixed point problems and variational inequalities. Fixed Point Theory Appl. 2013, 121 (2013)

43. Xu, HK: Iterative algorithms for nonlinear operators. J. Lond. Math. Soc. 66, 240-256 (2002)

44. Yang, H, Zhou, L, Li, Q: A parallel projection method for a system of nonlinear variational inequalities. Appl. Math. Comput. 217, 1971-1975 (2010)

45. Yao, Y, Cho, YJ, Liou, YC: Iterative algorithms for hierarchical fixed points problems and variational inequalities. Math. Comput. Model. 52(9-10), 1697-1705 (2010)

10.1186/1687-1812-2014-22

Cite this article as: Bnouhachem: A modified projection method for a common solution of a system of variational inequalities, a split equilibrium problem and a hierarchical fixed-point problem. Fixed Point Theory and Applications 2014, 2014:22

\section{Submit your manuscript to a SpringerOpen ${ }^{\odot}$ journal and benefit from:}

- Convenient online submission

- Rigorous peer review

Immediate publication on acceptance

- Open access: articles freely available online

- High visibility within the field

- Retaining the copyright to your article 